\title{
General One-Step Synthesis of Free Hexofuranosyl 1-Phosphates Using Unprotected 1-Thioimidoyl Hexofuranosides
}

\author{
Ronan Euzen, Vincent Ferrières,* and Daniel Plusquellec \\ Ecole Nationale Supérieure de Chimie de Rennes, UMR CNRS 6052 Synthèses et Activations de \\ Biomolécules, Institut de Chimie de Rennes, Avenue du Général Leclerc, F-35700 Rennes, France \\ vincent.ferrieres@ensc-rennes.fr
}

Received August 27, 2004 (Revised Manuscript Received October 21, 2004)

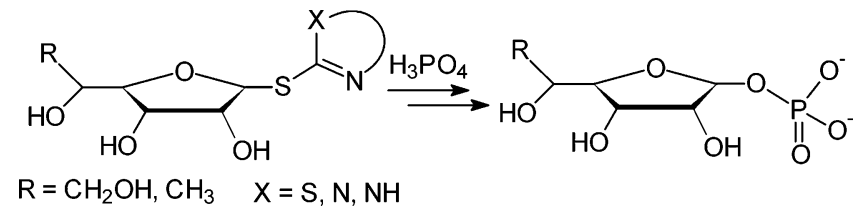

A general one-step strategy is developed for the synthesis of hexofuranosyl 1-phosphates starting from new unprotected glycofuranosyl donors. It required first the preparation of new 1-thiohexofuranosides bearing a thioimidoyl heterocycle as a leaving group. The presence of sulfur and/or nitrogen atom(s) on the aglycon allowed remote activation of these thioglycofuranosides by anhydrous phosphoric acid and led to the target phosphates $9,27,29$, and 30 in good to excellent selectivities and, more importantly, with very limited or no ring expansion. Moreover, this onestep phosphorylation reaction could be significantly improved by avoiding any tedious protecting group manipulations on negatively charged compounds and by focusing on a simple but general procedure of purification. This approach was applied to the diastereocontrolled synthesis of D-galactoand D-glucofuranosyl 1-phosphates and also to the preparation of rare epimer and/or deoxy counterparts, that is, D-manno- and D-fucofuranosyl derivatives.

\section{Introduction}

An explosive number of discoveries in the field of glycobiology over the past three decades have resulted in a better understanding of the roles of carbohydratebased biomolecules. ${ }^{1}$ These findings were possible since efficient purification procedures and analytical methods have been developed. Among the majority of natural glycoconjugates, it was shown that pentoses are equally found in a furanose or in a pyranose configuration, while hexoses generally exist in a pyranose form. However, the striking presence of hexofuranosyl entities was established in conjugates isolated from protozoae, ${ }^{2}$ fungi, ${ }^{3}$ bacteria, ${ }^{4}$ and archaebacteria. ${ }^{5} \mathrm{~A}$ well-known example is connected with the cellular wall of the causative agent of tuberculosis. Indeed, Mycobacterium tuberculosis is able to biosynthesize a highly complex membrane notably

(1) (a) Weymouth-Wilson, A. C. Nat. Prod. Rep. 1997, 14, 99-110. (b) Dwek, R. A. Chem. Rev. 1996, 96, 683-720.

(2) de Lederkremer, R. M.; Colli, W. Glycobiology 1995, 5, 547-552.

(3) Ayer, W. A.; Kawahara, N. Tetrahedron Lett. 1995, 36, 79537956.

(4) Nagaoka, M.; Hashimoto, S.; Shibata, H.; Kimura, I.; Sawada, H. Yokohura, T. Carbohydr. Res. 1996, 281, 285-291.

(5) (a) Benvegnu, T.; Brard, M.; Plusquellec, D. Curr. Opin. Colloid Interface Sci. 2004, 8, 469-479. (b) Koga, Y.; Nishihara, M.; Morii, H.; Akagawa-Matsushita, M. Microbiol. Rev. 1993, 57, 164-182. (c) Sprott, G. D. J. Bioenerg. Biomembr. 1992, 24, 555-566. containing an arabinogalactan characterized by the presence of five-membered rings for both $\alpha-/ \beta$-L-arabinofuranose and $\beta$-D-galactofuranose (D-Galf). ${ }^{6}$ On the other hand, Eubacterium saburreum, a Gram-positive bacteria frequently isolated from human dental plaques, produces a carbohydrate-based antigen with unique sugar composition since the linear chain of heptose is branched with D-fucofuranosyl (D-Fucf) units characterized by $\alpha$-anomeric configuration. ${ }^{7}$ It is also interesting to note that D-glucose (D-Glc) and its 2-epimer D-mannose (D-Man) were found as furanosyl residues in a rare nucleotidesugar Agrocine $84^{8}$ and in a polymannan produced by the oak lichen Evernia prunastri, ${ }^{9}$ respectively. Since all of these hexofuranosides are exclusively found in lower organism components, this offers novel opportunities for designing new pharmacophores and molecular probes for enzymatic studies. 63.

(6) Brennan, P. J.; Nikaido, H. Annu. Rev. Biochem. 1995, 64, 29-

(7) (a) Sato, N.; Nakzawa, F.; Ito, T.; Hishino, T.; Hoshino, E. Carbohydr. Res. 2003, 338, 923-930. (b) Sato, N.; Nakazawa, F.; Sato, M.; Hoshino, E.; Ito, T. Carbohydr. Res. 1993, 245, 105-111.

(8) (a) Moriguchi, T.; Wada, T.; Sekine, M. J. Org. Chem. 1996, 61 9223-9228. (b) Thompson, R. J.; Hamilton, R. H.; Pootjes, C. F. Antimicrob. Agents Chemother. 1979, 16, 293-296.

(9) Mićović, V. M.; Hranisavljević-Jakovljević, M.; Miljković-Stojanović, J. Carbohydr. Res. 1969, 10, 525-533. 
The growing interest in glycoconjugates must also be due to findings linked to pathways by which glycofuranoconjugates and polyhexofuranosides are biosynthesized and/or metabolized. Three families of enzymes have been identified: some galactofuranosidases, ${ }^{10}$ a galactofuranosyltransferase, ${ }^{11}$ and the uridine diphosphogalactopyranose (UDP-Galp) mutase. ${ }^{11,12}$ This mutase, which was cloned and expressed from four bacteria strains, is the only enzyme capable for catalyzing the galactose ring contraction from UDP-Galp into a less-stable nucleotidegalactofuranose (UDP-Galf). However, to the best of our knowledge, no data dealing with the incorporation of D-Glc $f$, D-Manf, and D-Fuc $f$ entities are published so far. These observations explain why current efforts for a better understanding of the UDP-Gal $p$ mutase are mainly placed in the preparation of UDP-Galf. Since enzymatic approaches could afford limited amounts of this target, ${ }^{12 c, 13}$ chemical synthesis has also been reported by a few groups. All of them rely on a coupling between D-Gal $f$ 1-phosphate ${ }^{14}$ and an activated uridine monophosphate (UMP) derivative. ${ }^{12 b, 15,16}$ Along with these contributions, Thorson and co-workers ${ }^{17}$ proposed a quite general method for the synthesis of UDP- and TDP-nucleotidesugars using an $\alpha$-D-glucosyl phosphate thymidylyltransferase as a biocatalyst and hexosyl 1-phosphates as substrates, but in a pyranose configuration. Nevertheless, these studies open great opportunities for new applications of hexosyl 1-phosphates in chemoenzymatic synthesis.

In this global context, our laboratory has initiated a program dedicated to chemical synthesis of various hexofuranosyl-containing derivatives, ${ }^{18}$ that is, oligosaccharides, glycolipids, and anomeric phosphates. A chemical preparation of these hexofuranosyl 1-phosphates has to take into account their thermal and chemical sensitivity. Consequently, because protecting group interchange

(10) (a) Marino, C.; Herczegh, P.; de Lederkremer, R. M. Carbohydr. Res. 2001, 333, 123-128. (b) Chiocconi, A.; Marino, C.; de Lederkremer R. M. Carbohydr. Res. 2000, 323, 7-13. (c) Marino, C.; Marino, K.; Miletti, L.; Manso Alves, M. J.; de Lederkremer, R. M. Glycobiology 1998, 8, 901-904.

(11) Houseknecht, J. B.; Lowary, T. L. Curr. Opin. Chem. Biol. 2001 $5,677-682$.

(12) (a) Zhang, Q.; Liu, H. W. J. Am. Chem. Soc. 2001, 123, 67566766. (b) Zhang, Q.; Liu, H. W. J. Am. Chem. Soc. 2000, 122, 90659070. (c) Barlow, J. N.; Blanchard, J. S. Carbohydr. Res. 2000, 328, 473-480. (d) Barlow, J. N.; Girvin, M. E.; Blanchard, J. S. J. Am. Chem. Soc. 1999, 121, 6968-6969. (e) Lee, R. E.; Smith, M. D.; Nash, R. J.; Griffiths, R. C.; McNeil, M.; Grewal, R. K.; Yan, W.; Besra, G. S.; Brennan, P. J.; Fleet, G. W. J. Tetrahedron Lett. 1997, 38, 67336736. (f) Nassau, P. M.; Martin, S. L.; Brown, R. E.; Weston, A.; Monsey, D.; McNeil, M. R.; Duncan, K. J. Bacteriol. 1996, 178, 10471052.

(13) Lee, R.; Monsey, D.; Wetson, A.; Duncan, K.; Rithner, C.; McNeil, M. Anal. Biochem. 1996, 242, 1-7.

(14) (a) de Lederkremer, R. M.; Nahmad, V. B.; Varela, O. J. Org. Chem. 1994, 59, 690-692. (b) Chittenden, J. F. Carbohydr. Res. 1972, $25,36-41$.

(15) Tsvetkov, Y. E.; Nikolaev, A. V. J. Chem. Soc., Perkin Trans. 1 2000, 889-891.

(16) Marlow, A. L.; Kiessling, L. Org. Lett. 2001, 3, 2517-2519.

(17) (a) Jiang, J.; Biggins, J. B.; Thorson, J. S. Angew. Chem., Int. Ed. 2001, 40, 1502-1505. (b) Jiang, J.; Biggins, J. B.; Thorson, J. S. J. Am. Chem. Soc. 2000, 122, 6803-6804.

(18) (a) Gelin, M.; Ferrières, V.; Lefeuvre, M.; Plusquellec, D. Eur. J. Org. Chem. 2003, 1285-1293. (b) Ferrières, V.; Roussel, M.; Gelin M.; Plusquellec, D. J. Carbohydr. Chem. 2001, 20, 855-865. (c) Gelin, M.; Ferrières, V.; Plusquellec, D. Eur. J. Org. Chem. 2000, 1423-1431. (d) Velty, R.; Benvegnu, T.; Gelin, M.; Privat, E.; Plusquellec, D. Carbohydr. Res. 1997, 299, 7-14. (e) Ferrières, V.; Blanchard, S.; Fischer, D.; Plusquellec, D. Bioorg. Med. Chem. Lett. 2002, 12, 35153518. is often tedious on highly charged molecules, it may be judicious to introduce the phosphate group directly on unprotected substrates. However, such a strategy necessarily involves a phosphorylation reaction preserving the five-membered ring of the starting materials. Finally, one must keep in mind that one of the drawbacks in carbohydrate-containing derivatives still remains in the stereocontrol of the coupling reactions, more particularly within the furanose series in which competition between anomeric effects and steric ones are generally observed..$^{19}$ These main requirements were fulfilled by the following: (i) remote activation of new unprotected thioimidoyl hexofuranosides by dry phosphoric acid, and (ii) careful and general isolation procedure. We present herein the full details of synthesis of the thiofuranosidic precursors and the desired D-Galf 1-phosphate as a model target. The proposed methodology was further successfully extended to the corresponding D-Glc $f$ and D-Man $f$ derivatives, and also to D-Fucf 1-phosphate, described here for the first time.

\section{Results and Discussion}

On the basis of the chemical structure of UDP-Galp, and considering the key points previously disclosed, we focused on developing a one-step chemical synthesis of hexofuranosyl 1-phosphates characterized by a 1,2-cis stereochemistry. Despite considerable progress in glycochemistry, the preparation of 1,2-cis glycosides still remains a challenge of particular interest. ${ }^{20}$ In this context, Hanessian has proposed the attractive concept of the remote activation process. ${ }^{21}$ While the best results were obtained with the 3-methoxy-2-pyridyloxy (MOP) group, orthogonal glycosylation was recently demonstrated by Demchenko using 2-O-benzylated $S$-benzoxazolyl ${ }^{22}$ or $S$-thiazolyl glycosides. ${ }^{23}$ In the present study, we investigated the electrofugal character of protonated aromatic aglycons belonging to the family of substituted thioimidoyl derivatives containing a third heteroatom $(\mathrm{S}$ or $\mathrm{N}$ ) suitably placed to modulate the basicity of the hard center. Then we first evaluated the feasibility of this concept for the synthesis of the relevant Galf 1-phosphate. The desired $S$-galactosides $\mathbf{5}-\mathbf{7}$ were readily and exclusively obtained from known penta- $O$-acetyl galactofuranose $(\mathbf{1})^{24}$ and 2-mercaptobenzimidazole, 2-mercaptobenzothiazole, or 2-mercaptopyrimidine, respectively, upon activation by boron trifluoride etherate complex (Scheme 1). While an anomeric mixture was obtained for the benzothiazolyl derivative 3 ( $\beta / \alpha, 6.7: 1)$, complete $\beta$-stereodirection was observed for benzimidazolyl and pyrimidinyl furanosides $\mathbf{2}$ and $\mathbf{4}$. Subsequent deacylation under basic Zémplen transesterification or in a hydroalcoholic solution of triethylamine afforded the desired thiofuranosides 5-7.

(19) Kirby, A. J. In The Anomeric Affect and Related Stereoelectronic Effects at Oxygen; Springer-Verlag: Berlin, Heidelberg, New York, 1983.

(20) Demchenko, A. V. Curr. Org. Chem. 2003, 7, 35-79.

(21) Hanessian, S.; Lou, B. Chem. Rev. 2000, 100, 4443-4463.

(22) (a) Demchenko, A. V.; Malysheva, N. N.; De Meo, C. Org. Lett. 2003, 5, 455-458. (b) Demchenko, A. V.; Kamat, M. N.; De Meo, C. Synlett 2003, 1287-1290.

(23) Demchenko, A. V.; Pornsuiyasak, P.; De Meo C.; Malysheva, N. N. Angew. Chem., Int. Ed. 2004, 43, 3069-3072.

(24) Ferrières, V.; Gelin, M.; Boulch, R.; Toupet, L.; Plusquellec, D. Carbohydr. Res. 1998, 314, 79-83. 
SCHEME 1. Synthesis of Galactofuranosyl Donors $5,6,7^{a}$

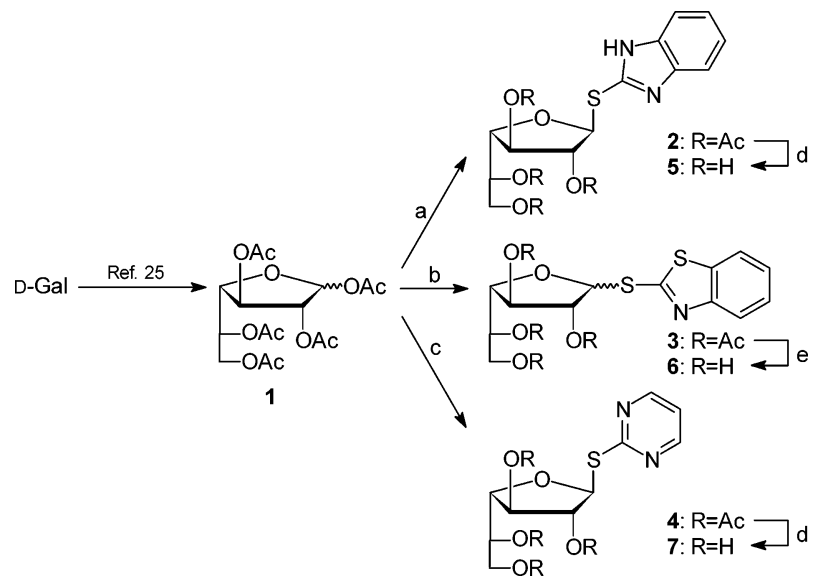

${ }^{a}$ Reagents and conditions: (a) 2-mercaptobenzimidazole, $\mathrm{BF}_{3} \cdot \mathrm{Et}_{2} \mathrm{O}$, $\mathrm{CH}_{2} \mathrm{Cl}_{2}$ (91\%); (b) 2-mercaptobenzothiazole, $\mathrm{BF}_{3} \cdot \mathrm{OEt}_{2} \mathrm{O}, \mathrm{CH}_{2} \mathrm{Cl}_{2}$ [83\% ( $\alpha / \beta, 1: 6.7)]$; (c) 2-mercaptopyrimidine, $\mathrm{BF}_{3} \cdot \mathrm{OEt}_{2} \mathrm{O}, \mathrm{CH}_{2} \mathrm{Cl}_{2}$ (99\%); (d) $\mathrm{MeONa}, \mathrm{MeOH}$ (5: 100\%; 7: 98\%); (e) $\mathrm{MeOH}, \mathrm{H}_{2} \mathrm{O}, \mathrm{NEt}_{3}$ $[67 \%(\alpha / \beta, 1: 6.7)]$.

With these galactofuranosyl donors in hand, phosphorylation was first monitored by ${ }^{1} \mathrm{H}$ NMR spectroscopy using $N, N$-dimethylformamide- $d_{7}$ as a solvent. Quite surprisingly, the pyrimidinyl donor $\mathbf{7}$ was proved to be highly stable under such conditions since neither phosphorylation, degradation, nor anomerization occurred. Nevertheless, the thiogalactofuranoside 5, with a benzimidazolyl aglycon, was smoothly activated by phosphoric acid (Figure 1). The resulting data indicated that $\mathbf{5}$ was completely consumed after only $18 \mathrm{~min}$ at room temperature and was indeed converted into the target phosphate 8 with less than $10 \%$ of released D-galatose, even after prolonged reaction times. ${ }^{1} \mathrm{H}$ NMR spectra unambiguously showed characteristic data relevant for furanosyl phosphates and notably suitable multiplicity for H-1 signals significantly different from that observed for their pyranosyl counterparts. ${ }^{25,26}$ Indeed, compound $8 \alpha$ is characterized by a doublet of a doublet with similar $J_{\mathrm{H}-1, \mathrm{P}}$ and $J_{\mathrm{H}-1, \mathrm{H}-2}$ values near $3.1 \mathrm{~Hz}$ at $5.54 \mathrm{ppm}$, while the trans relationship between $\mathrm{H}-1$ and $\mathrm{H}-2$ in $\mathbf{8} \beta$ was deduced from a doublet with small $J_{\mathrm{H}-1, \mathrm{H}-2}(<1 \mathrm{~Hz})$ but higher $J_{\mathrm{H}-1, \mathrm{P}}$ coupling constant $(5.4 \mathrm{~Hz})$ at $5.58 \mathrm{ppm}$. The monitoring also revealed the faster formation of the desired $\alpha$-D-Gal $f$ 1-phosphate $\mathbf{8} \alpha$. This result, therefore, supports a mechanism involving protonation of the $\beta$-leaving group followed by a nucleophilic attack by the released oxyanion on the $\alpha$-face. However, $8 \alpha$ slowly anomerized into the less hindered $8 \beta$ so that the $\alpha / \beta$ ratio reached $0.6: 1$ at equilibrium.

The reaction was also carried out using benzothiazolyl donor 6. A longer reaction time $\left(13 \mathrm{~h}\right.$ at $\left.20{ }^{\circ} \mathrm{C}\right)$ resulted in lower overall conversion yield and in poorer $\alpha$-selectivity. Interpretation of phosphorylation results from furanosides 5-7 could not rely only on the $\mathrm{p} K_{\mathrm{a}}$ values of the leaving groups. ${ }^{21}$ Nevertheless, as previously described in the pyranose series, ${ }^{21,27}$ the respective reactivities of

(25) Hanessian, S.; Lu, P. H. Ishida, H. J. Am. Chem. Soc. 1998 , 120, 13296-13300

(26) O’Connor, J. V.; Nunez, H. A.; Barker, R. Biochemistry 1979, $18,500-507$. these unprotected donors correlate well with the observed chemical shifts of $\mathrm{H}-1$ : the lower the chemical shift (5: $\left.\delta_{\mathrm{H}-1} 5.75 \mathrm{ppm}, \mathbf{6} \beta: \delta_{\mathrm{H}-1} 6.00 \mathrm{ppm}, \mathbf{7}: \delta_{\mathrm{H}-1} 6.20 \mathrm{ppm}\right)$, the more reactive is the glycosyl donor.

To demonstrate the applicability of this methodology, we further focused on a purification protocol that does not alter the desired product, neither the ring size nor the $\alpha / \beta$ ratio. Excess of phosphoric acid was first eliminated as barium phosphate by adjusting the $\mathrm{pH}$ between 8 and 9 with a saturated solution of barium hydroxide (Scheme 2). After centrifuging, the supernatant liquid was carefully neutralized using a strong acidic resin so that the phosphosugar remained as a barium salt. Freeze-drying followed by selective removal of released 2-mercaptobenzimidazole gave the desired phosphate, however, along with D-Gal. Removal of the reducing carbohydrate required resin-assisted barium/ammonium metathesis and an ionic exchange chromatography using an anionic column $\left(\mathrm{CO}_{3}{ }^{2-}\right.$-form $)$. The crucial point of this last step relied on a judicious choice of the eluting solution since the target phosphate $\mathbf{9}$ is highly sensitive in aqueous solution at $\mathrm{pH}$ below 7. In our hands, first attempts of chromatographic purification using a molar ammonium hydroxide aqueous solution unfortunately resulted in detrimental degradation of $\mathbf{9}$ due to a toolong retaining period. However, the ionic strength of the eluant was advantageously increased by using an ammonium carbonate aqueous solution, which was easily freeze-dried after elution. As a consequence, galactofuranosyl 1-phosphate $\mathbf{9}$ was synthesized and isolated in a $58 \%$ yield as a 1.2:1 anomeric mixture of $\mathbf{9} \alpha$ and $\mathbf{9} \beta$, characterized by NMR data similar to that of ammonium and cyclohexylammonium derivatives. ${ }^{14 a}$ While this stereoselectivity is slightly lower than that previously described using a furanosyl bromide as a donor, our methodology complements well that of de Lederkremer ${ }^{14 a}$ since no protecting-group manipulation is required for further use of the target phosphate. This approach is also characterized by a shortened three-step synthetic scheme and so resulted in a better overall yield. Indeed, phosphate 9 was obtained in 53\% overall yield from the pentacetyl galactofuranose (10).

Encouraged by this successful result, this approach was extended to the synthesis of furanosyl 1-phosphate corresponding to D-Glcf, D-Manf, and D-Fucf. The benzimidazolyl gluco- and mannofuranosides $\mathbf{1 2}$ and $\mathbf{1 5}{ }^{28}$ were obtained from $\mathbf{1 0}$ and $\mathbf{1 3},{ }^{24}$ respectively, according to the same procedure as in the galactose series (Scheme 3). Considering the low availability of D-fucose, preparation of fucosyl donor $\mathbf{2 6}$ involved a multistep synthesis starting from $n$-octyl galactofuranoside (16) ${ }^{29}$ (Scheme 4$)$. The primary hydroxyl was first selectively tritylated using freshly prepared $4-N, N$-(dimethylamino)- $1-N$-triphenylmethylpyridinium chloride. ${ }^{30}$ Subsequent benzylation,

(27) (a) Zhang, Z.; Ollman, I. R.; Ye, X. S.; Wischnat, R.; Baasov, T.; Wong, C. H. J. Am. Chem. Soc. 1999, 121, 734-753. (b) Douglas, N. L.; Ley, S. V.; Lucking, U.; Warriner, S. L. J. Chem. Soc., Perkin Trans. 1 1998, 51-65.

(28) It is interesting to note that $\mathrm{H}-1$ is characterized by a complex and unusual signal assigned by ${ }^{1} \mathrm{H}$ NMR. Indeed, because signals from H-2 and H-3 are superimposed, second-order effects arose out of virtual long-range coupling and so resulted in producing a more complex signal (six peaks) than the expected doublet. See, for example: Kotowycz, G.; Lemieux, R. U. Chem. Rev. 1973, 73, 669-698.

(29) Ferrières, V.; Bertho, J. N.; Plusquellec, D. Carbohydr. Res. 1998, $311,25-35$. 


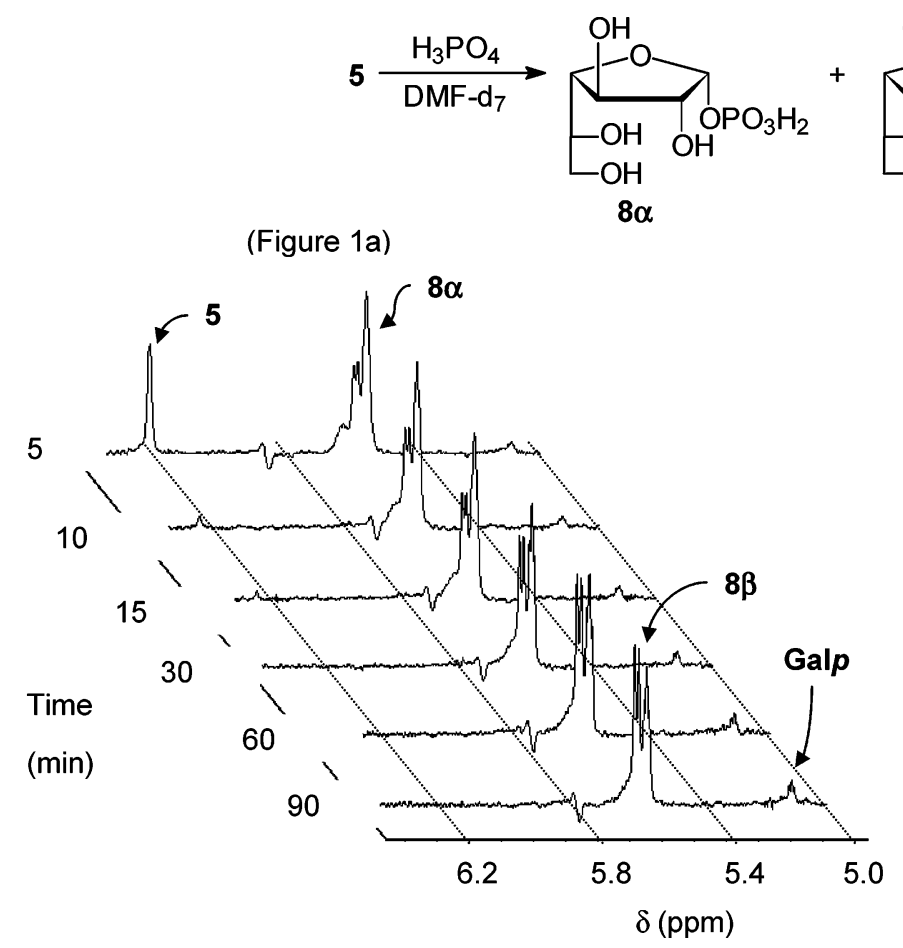

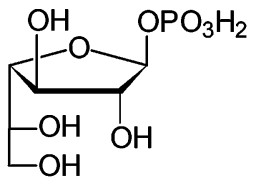

$8 \beta$

(Figure 1b)

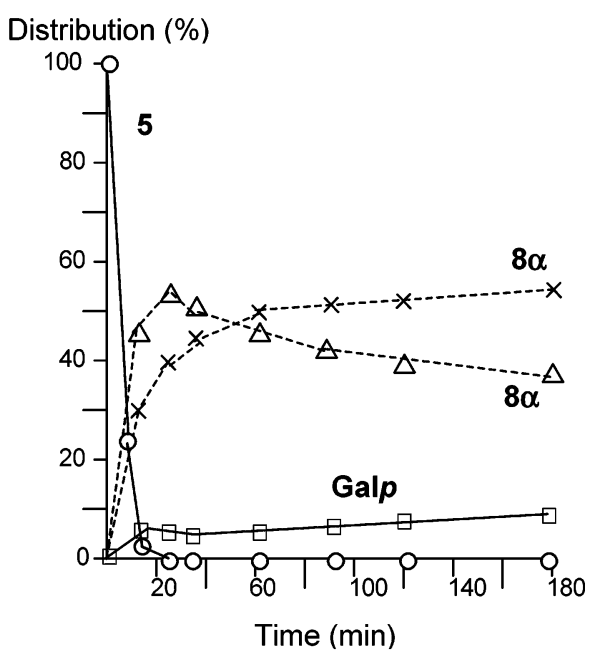

FIGURE 1. Phosphorylation of donor 5: (a) Selected ${ }^{1} \mathrm{H}$ NMR data (H-1 area); (b) distribution $(\bigcirc \mathbf{5}, \times \mathbf{8} \boldsymbol{\alpha}, \Delta \mathbf{8} \boldsymbol{\beta}, \square$ Galp) vs reaction time.

\section{SCHEME 2. Synthesis of D-Galactofuranosyl} 1-Phosphate $9 \alpha, \beta^{a}$

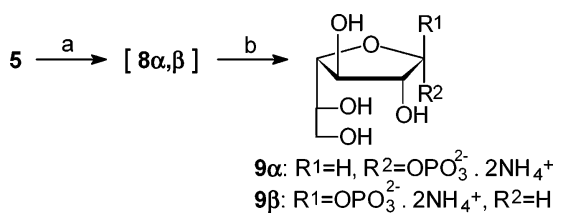

${ }^{a}$ Reagents and conditions: (a) $\mathrm{H}_{3} \mathrm{PO}_{4}, \mathrm{DMF}, 18 \mathrm{~min}$; (b) $\mathrm{Ba}(\mathrm{OH})_{2} ; \mathrm{IR}-120\left(\mathrm{H}^{+}\right.$-form); $\mathrm{EtOH}_{\mathrm{abs}}$, AcOEt (v/v 1:6); IR-120 $\left(\mathrm{NH}_{4}{ }^{+}\right.$-form); Amberlyst A-26 $\left(\mathrm{CO}_{3}{ }^{2-}\right.$-form) [58\% overall yield $(\alpha / \beta, 1.2: 1)]$

\section{SCHEME 3. Synthesis of Thioglucoside 10 and Mannofuranosyl Donor $12^{a}$}

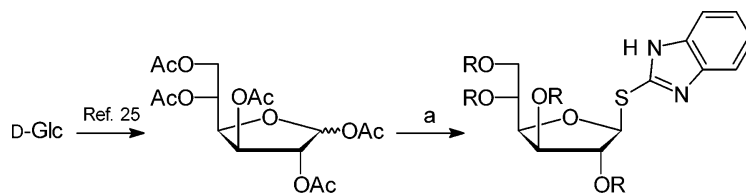

10 11: $\mathrm{R}=\mathrm{Ac} \longrightarrow \mathrm{B}$

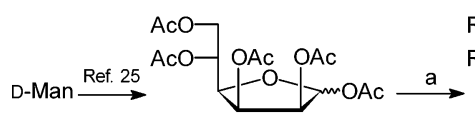

13

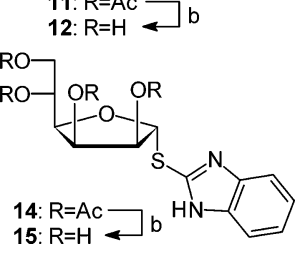

${ }^{a}$ Reagents and conditions: (a) 2-mercaptobenzimidazole, $\mathrm{BF}_{3} \cdot \mathrm{OEt}_{2}, \mathrm{CH}_{2} \mathrm{Cl}_{2}$ (11: 67\%; 14: 71\%); (b) MeONa, MeOH (12: $100 \%$; 15: $100 \%)$.

acidolysis, ${ }^{18 d, 31}$ and tosylation upon conventional procedures afforded 20, which was reduced by lithium alumi-

(30) Hernadez, O.; Chaudhary, S. K.; Cox, R. H.; Poter, J. Tetrahedron Lett. 1981, 22, 1491-1494.
SCHEME 4. Preparation of Fucofuranoside $\mathbf{2 6}^{\boldsymbol{a}}$

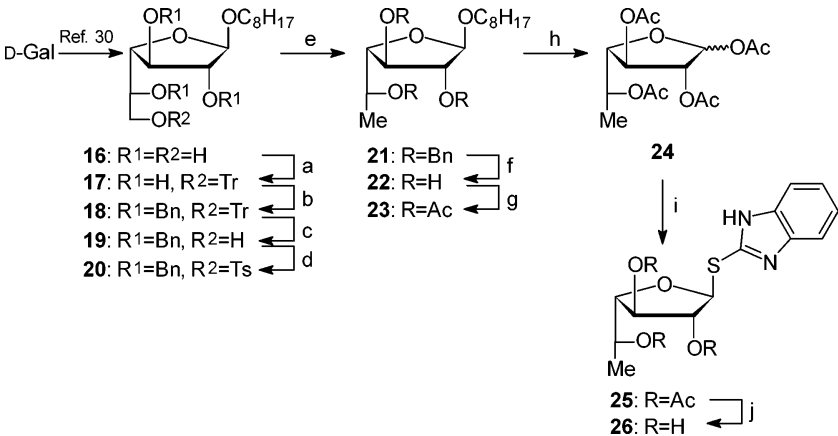

${ }^{a}$ Reagents and conditions: (a) 4-N,N-(dimethylamino)-1- $N$ triphenylmethylpyridinium chloride, $\mathrm{CH}_{2} \mathrm{Cl}_{2}(80 \%)$; (b) $\mathrm{BnBr}, \mathrm{NaH}$, DMF (99\%); (c) AcOH, $\mathrm{H}_{2} \mathrm{O}, 100{ }^{\circ} \mathrm{C}$ (78\%); (d) TsCl, DMAP, $\mathrm{NEt}_{3}$, $\mathrm{CH}_{2} \mathrm{Cl}_{2}$ (96\%); (e) $\mathrm{LiAlH}_{4}, \mathrm{Et}_{2} \mathrm{O}$ (90\%); (f) $\mathrm{H}_{2}, \mathrm{Pd}(\mathrm{OAc})_{2}, \mathrm{AcOH}$, AcOEt (100\%); (g) $\mathrm{Ac}_{2} \mathrm{O}$, pyridine (96\%); (h) $\mathrm{Ac}_{2} \mathrm{O}, \mathrm{H}_{2} \mathrm{SO}_{4}, \mathrm{CH}_{2} \mathrm{Cl}_{2}$ [94\% ( $\alpha / \beta, 1: 4)]$; (i) 2-mercaptobenzimidazole, $\mathrm{BF}_{3} \cdot \mathrm{OEt}_{2}, \mathrm{CH}_{2} \mathrm{Cl}_{2}$ (90\%); (j) $\mathrm{MeOH}, \mathrm{MeONa}(93 \%)$.

num hydride to yield the fucofuranoside 21. Protecting group interconversion followed by removal of the temporary protecting alkoxy chain under carefully controlled acetolysis further gave $\mathbf{2 4}^{32}$ in $48 \%$ overall yield for eight steps. The latter intermediate was converted into donor 26 through treatment with 2-mercaptobenzimidazole in the presence of boron trifluoride etherate complex and Zémplen deacetylation.

Subsequently, phosphorylation of these three donors 12,15 , and 26 was explored, and the reactions were

(31) Pathak, A. K.; Pathak, V.; Seitz, L.; Maddry, J. A.; Gurcha, S. S.; Besra, G. S.; Suling, W. J.; Reynolds, R. C. Bioorg. Med. Chem. 2001, 9, 3129-3143.

(32) Kinoshita, T.; Miwa, T.; Clardy, J. Carbohydr. Res. 1985, 143, 249-255. 
SCHEME 5. Synthesis of Furanosyl 1-Phosphates 27, 29, and $30^{a}$
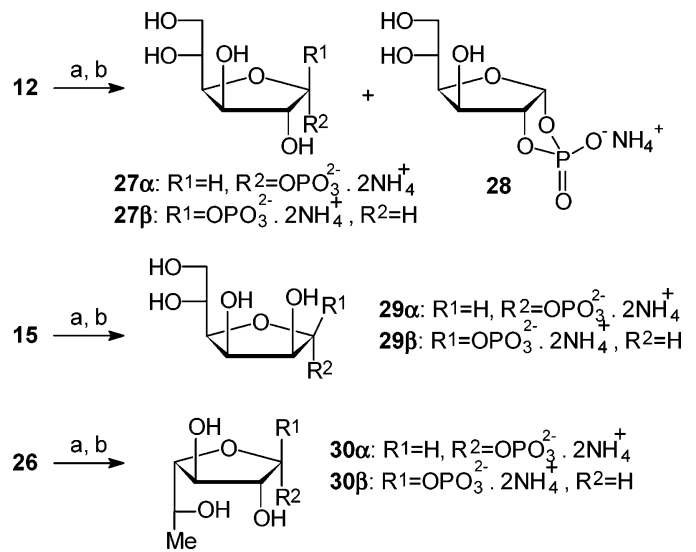

${ }^{a}$ Reagents and conditions: (a) $\mathrm{H}_{3} \mathrm{PO}_{4}, \mathrm{DMF}$ (27: 90 min; 29: $15 \mathrm{~min}$; 30: $20 \mathrm{~min})$; (b) purification procedure [27: $48 \%(\alpha / \beta, 1.2$ : 1), 28: $3 \%$; 29: $67 \%(\alpha / \beta, 1.6: 1)$; 30: $90 \%(\alpha / \beta, 1.5: 1)]$.

monitored by NMR to determine optimum reaction times. As expected, formation of the corresponding phosphates was found to be dependent on the hexose series. The reactivity of glucofuranoside $\mathbf{1 2}$, which led to $\mathbf{2 7}$ (Scheme 5 ), seemed to be lower than that of its 4-epimer $\mathbf{5}$ since $3.5 \mathrm{~h}$ were required to have good $\alpha$-stereoselectivity and high conversion yield. Assignment of the anomeric proton corresponding to $27 \beta$ was characterized by a doublet at $5.39 \mathrm{ppm}$ with a small coupling constant $J_{\mathrm{H}-1, \mathrm{H}-2}(<1 \mathrm{~Hz})$ and a value of $7.1 \mathrm{~Hz}$ for $J_{\mathrm{H}-1, \mathrm{P}}$, while $27 \alpha$ stood out from the $\beta$-anomer by a double doublet at $5.65 \mathrm{ppm}$ with a $J_{\mathrm{H}-1, \mathrm{H}-2}$ of $4.1 \mathrm{~Hz}$ and a distinct $J_{\mathrm{H}-1, \mathrm{P}}$ of $6.4 \mathrm{~Hz}$. Moreover, the NMR monitoring also showed the formation of a byproduct that appeared after $90 \mathrm{~min}$. Its chemical structure was established on the basis of significant chemical shifts and coupling constant values, that is, for $\mathrm{H}-1, \delta 6.00$ ppm with a $J_{\mathrm{H}-1, \mathrm{H}-2}$ of $4.1 \mathrm{~Hz}$, and for $\mathrm{P}, \delta$ $15.0 \mathrm{ppm}$ with a $J_{\mathrm{H}-1, \mathrm{P}}$ of $16.8 \mathrm{~Hz}$. These data are indicative of a strained bicyclic compound ${ }^{33}$ with $\alpha$-anomeric configuration and unambiguously correspond to the 1,2-cyclic phosphate 28. Formation of the latter can be rationalized by an intramolecular dehydration of firstformed $27 \alpha$ prior to anomerization of $27 \alpha$ into less hindered $27 \beta$. Therefore, to minimize this cyclization, the reaction was quenched after $90 \mathrm{~min}$ at room temperature. The previously disclosed purification protocol gave the target D-Glcf 1-phosphate 27 ( $\alpha / \beta$ 1.2:1) isolated in $48 \%$ yield, accompanied by cyclic phosphate $28(3 \%)$.

Within the D-mannose and D-fucose series, remote activation of $\mathbf{1 5}$ and $\mathbf{2 6}$ smoothly afforded $\mathbf{2 9}$ and $\mathbf{3 0}$, respectively, after only $15-20 \mathrm{~min}$ reaction times. The desired phosphates $\mathbf{2 9}$ and $\mathbf{3 0}$ were then isolated as ammonium salts in 66 and $90 \%$ yield, respectively (Scheme 5). Providing that ammonium carbonate was used in the eluting solution during the last ionic exchange chromatography, no byproduct was observed after purification. Anomeric configurations of 6-deoxy-galactofuranosyl derivatives $30 \alpha$ and $30 \beta$ were determined from significant NMR data close to that described for 9. For D-Manf 1-phosphate 29, the relationship between $\mathrm{H}-1$ and H-2 was based on the assumption of a lower-field signal

(33) Fathi, R.; Jordan, F. J. Org. Chem. 1986, 51, 4143-4146. for the trans-29 $\alpha\left(\delta_{\mathrm{C}-1} 102.7 \mathrm{ppm}\right)$ than that of $\mathbf{2 9} \beta\left(\delta_{\mathrm{C}-1}\right.$ $96.5 \mathrm{ppm}) .{ }^{24,34}$ The NMR monitoring of the phosphorylation of $\mathbf{2 6}$ revealed that the 1,2-cis phosphate was kinetically obtained in agreement with the proposed mechanism, followed by fast anomerization into the much more stable 1,2-trans counterpart. Moreover, it was observed that this epimerization also occurred during isolation of the desired ammonium salt. As a consequence, 29 was obtained as a $\alpha, \beta$ mixture slightly enriched in $\alpha$-anomer $(\mathbf{2 9} \alpha / \mathbf{2 9} \beta$ 1.6:1).

\section{Conclusions}

A highly general and concise synthesis of hexofuranosyl 1-phosphates was achieved using new unprotected hexofuranosyl donors. The strategy was based on the remote activation of readily available thioimidoyl furanosides by dry phosphoric acid. Our study on a set of galactofuranosides established that best results were obtained using $S$-benzimidazolyl aglycon as a leaving group. It is particularly important to note that the furanoid ring of the substrates was generally not altered under reaction conditions nor during the purification steps so that furanosyl 1-phosphates were prepared in good to excellent yields and in interesting 1,2-cis stereoselectivities. As a consequence, this approach not only allowed the synthesis of known D-Galf 1-phosphate, D-Glcf, and D-Manf epimers but also, to the best of our knowledge, afforded for the first time the corresponding D-Fucf derivative. This, therefore, opens a very interesting opportunity for an easier access to rare nucleotidehexofuranoses that are crucial products for biological studies of glycofuranoconjugates.

\section{Experimental Section}

General Procedure A for the Synthesis of Acetylated Thiofuranosides. To a solution of per- $O$-acetylated hexofuranose $(5 \mathrm{mmol})$ in anhyd dichloromethane $(80 \mathrm{~mL})$ were successively added the thione $(15 \mathrm{mmol})$ and $\mathrm{BF}_{3} \cdot \mathrm{OEt}_{2}(45$ $\mathrm{mmol}$ ). The mixture was stirred for $24 \mathrm{~h}$ at the appropriate temperature then diluted with dichloromethane $(80 \mathrm{~mL})$, and washed successively with a saturated solution of aq $\mathrm{NaHCO}_{3}$ $(3 \times 50 \mathrm{~mL})$ and water $(3 \times 100 \mathrm{~mL})$. The aqueous layers thus obtained were extracted with dichloromethane $(3 \times 50 \mathrm{~mL})$, and the combined organic layers were dried $\left(\mathrm{MgSO}_{4}\right)$ and finally concentrated. Purification of the residue by flash chromatography on silica gel afforded the desired thioglycofuranoside.

2-Benzimidazolyl 2,3,5,6-Tetra- $O$-acetyl-1-thio- $\beta$-D-galactofuranoside (2). This compound was synthesized according to the general procedure A from $1(2 \mathrm{~g}, 5.12 \mathrm{mmol})$ at room temperature. Chromatographic purification (light petroleum/ EtOAc, $1: 1)$ gave $2(2.25 \mathrm{~g}, 91 \%)$ as a colorless oil: $R_{f} 0.3$ (light

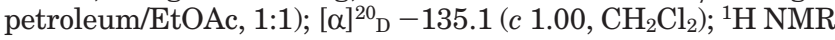
$\left(400 \mathrm{MHz}, \mathrm{CDCl}_{3}\right) \delta 2.05,2.09,2.11,2.13\left(4 \mathrm{~s}, 12 \mathrm{H}, \mathrm{CH}_{3} \mathrm{CO}\right)$, 4.16 (dd, $1 \mathrm{H}, J=5.8,11.7 \mathrm{~Hz}, \mathrm{H}-6 \mathrm{a}), 4.49$ (dd, $1 \mathrm{H}, J=3.8$, $5.4 \mathrm{~Hz}, \mathrm{H}-4), 4.55$ (dd, $1 \mathrm{H}, J=5.8,11.7 \mathrm{~Hz}, \mathrm{H}-6 \mathrm{~b}), 5.13$ (dd, $1 \mathrm{H}, J=2.3,5.4 \mathrm{~Hz}, \mathrm{H}-3), 5.32(\mathrm{t}, 1 \mathrm{H}, J=2.3 \mathrm{~Hz}, \mathrm{H}-2), 5.37$ (td, $1 \mathrm{H}, J=3.8,5.8 \mathrm{~Hz}, \mathrm{H}-5), 5.94(\mathrm{~d}, 1 \mathrm{H}, J=2.0 \mathrm{~Hz}, \mathrm{H}-1)$, 7.23-7.27 (m, 2H, H-6', H-7'), 7.47-7.71 (m, 2H, H-5', H-8'), 10.41 (br s, $1 \mathrm{H}, \mathrm{NH}) ;{ }^{13} \mathrm{C} \mathrm{NMR}\left(100 \mathrm{MHz}, \mathrm{CDCl}_{3}\right) \delta 20.7,20.7$, 20.9, $20.9\left(\mathrm{CH}_{3} \mathrm{CO}\right), 61.6(\mathrm{C}-6), 68.9(\mathrm{C}-5), 76.5$ (C-3), 80.9, 80.9 (C-2, C-4), 89.2 (C-1), 110.7, 119.2 (C-5', C-8'), 123.2 (C-6', C-7'),

(34) For methyl D-mannofuranosides and protected derivatives, see also: Bock, K.; Pedersen, C. Adv. Carbohydr. Chem. Biochem. 1983, $41,27-66$. 
145.6 (C-2', C-4', C-9'), 169.2, 169.9, 170.1, 171.1 (C=O); HRMS $\left(\mathrm{ESI}^{+}\right) \mathrm{m} / z$ calcd for $\mathrm{C}_{21} \mathrm{H}_{24} \mathrm{~N}_{2} \mathrm{NaO}_{9} \mathrm{~S}[\mathrm{M}+\mathrm{Na}]^{+} 503.1100$, found 503.1100. Anal. calcd for $\mathrm{C}_{21} \mathrm{H}_{24} \mathrm{~N}_{2} \mathrm{O}_{9} \mathrm{~S}$ : C, 52.49; $\mathrm{H}, 5.03 ; \mathrm{N}$, 5.83 ; S, 6.67. Found: C, 52.37; H, 5.01; N, 5.69; S, 6.47.

2-Benzothiazolyl 2,3,5,6-Tetra-O-acetyl-1-thio-D-galactofuranoside (3). This compound was prepared as described in general procedure A from $1(50 \mathrm{mg}, 0.13 \mathrm{mmol})$ at room temperature. Chromatographic purification $\left(\mathrm{CH}_{2} \mathrm{Cl}_{2} /\right.$ EtOAc, 95:5) gave an anomeric mixture $(\alpha / \beta, 1: 6.7)$ of $\mathbf{3}$ (53 mg, $83 \%)$ as a yellow oil: $R_{f} 0.5\left(\mathrm{CH}_{2} \mathrm{Cl}_{2} / \mathrm{EtOAc}, 9: 1\right) .3 \alpha:{ }^{1} \mathrm{H}$ NMR $\left(400 \mathrm{MHz}, \mathrm{CDCl}_{3}\right) \delta 2.01,2.09,2.10,2.11(4 \mathrm{~s}, 12 \mathrm{H}$, $\mathrm{CH}_{3} \mathrm{CO}$ ), 4.14 (dd, $\left.1 \mathrm{H}, J=6.8,12.0 \mathrm{~Hz}, \mathrm{H}-6 \mathrm{a}\right), 4.21-4.24(\mathrm{~m}$, $1 \mathrm{H}, \mathrm{H}-4), 4.37$ (dd, $1 \mathrm{H}, J=4.2,12.0 \mathrm{~Hz}, \mathrm{H}-6 \mathrm{~b}), 5.32-5.38(\mathrm{~m}$, $2 \mathrm{H}, \mathrm{H}-3, \mathrm{H}-5), 5.63$ (dd, $1 \mathrm{H}, J=4.3,5.6 \mathrm{~Hz}, \mathrm{H}-2), 6.55$ (d, $1 \mathrm{H}$, $J=5.6 \mathrm{~Hz}, \mathrm{H}-1), 7.32\left(\mathrm{td}, 1 \mathrm{H}, J=1.0,8.4 \mathrm{~Hz}, \mathrm{H}-6^{\prime}\right), 7.42(\mathrm{td}$, $\left.1 \mathrm{H}, J=1.3,8.4 \mathrm{~Hz}, \mathrm{H}-7^{\prime}\right), 7.77\left(\mathrm{dd}, 1 \mathrm{H}, J=1.0,8.4 \mathrm{~Hz}, \mathrm{H}-8^{\prime}\right)$, $7.90\left(\mathrm{dd}, 1 \mathrm{H}, J=1.3,8.4 \mathrm{~Hz}, \mathrm{H}-5^{\prime}\right) ;{ }^{13} \mathrm{C} \mathrm{NMR}\left(100 \mathrm{MHz}, \mathrm{CDCl}_{3}\right)$ $\delta$ 20.5, 20.6, 20.8, $21.0\left(\mathrm{CH}_{3} \mathrm{CO}\right), 62.4(\mathrm{C}-6), 69.3(\mathrm{C}-5), 75.5$ (C-3), 76.3 (C-2), 81.2 (C-4), 86.9 (C-1), 121.1 (C-5'), 122.1

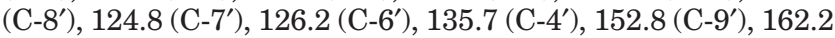
$\left(\mathrm{C}-2^{\prime}\right), 169.3,169.6,169.9,170.4(\mathrm{C}=\mathrm{O}) .3 \beta$ : ${ }^{1} \mathrm{H}$ NMR $(400$ $\left.\mathrm{MHz} \mathrm{CDCl}_{3}\right) \delta 1.98,2.10,2.11,2.13\left(4 \mathrm{~s}, 12 \mathrm{H}, \mathrm{CH}_{3} \mathrm{CO}\right), 4.18$ (dd, $1 \mathrm{H}, J=6.9,11.8 \mathrm{~Hz}, \mathrm{H}-6 \mathrm{a}), 4.32$ (dd, $1 \mathrm{H}, J=4.3,11.8$ $\mathrm{Hz}, \mathrm{H}-6 \mathrm{~b}), 4.49$ (dd, $1 \mathrm{H}, J=4.3,5.3 \mathrm{~Hz}, \mathrm{H}-4), 5.13$ (dd, $1 \mathrm{H}, J$ $=2.0,5.3 \mathrm{~Hz}, \mathrm{H}-3), 5.36(\mathrm{t}, 1 \mathrm{H}, J=2.0 \mathrm{~Hz}, \mathrm{H}-2), 5.42(\mathrm{dt}, 1 \mathrm{H}$, $J=4.3,6.9 \mathrm{~Hz}, \mathrm{H}-5), 6.27(\mathrm{~d}, 1 \mathrm{H}, J=1.7 \mathrm{~Hz}, \mathrm{H}-1), 7.31(\mathrm{td}$, $\left.1 \mathrm{H}, J=1.0,7.9 \mathrm{~Hz}, \mathrm{H}-6^{\prime}\right), 7.41\left(\mathrm{td}, 1 \mathrm{H}, J=1.0,8.1 \mathrm{~Hz}, \mathrm{H}-7^{\prime}\right)$, $7.76\left(\mathrm{dd}, 1 \mathrm{H}, J=1.0,8.1 \mathrm{~Hz}, \mathrm{H}-8^{\prime}\right), 7.90(\mathrm{dd}, 1 \mathrm{H}, J=1.0,7.9$ $\left.\mathrm{Hz}, \mathrm{H}-5^{\prime}\right) ;{ }^{13} \mathrm{C} \mathrm{NMR}\left(100 \mathrm{MHz}, \mathrm{CDCl}_{3}\right) \delta 20.6,20.6,20.6,20.8$ $\left(\mathrm{CH}_{3} \mathrm{CO}\right), 62.4(\mathrm{C}-6), 69.0(\mathrm{C}-5), 76.4(\mathrm{C}-3), 80.9(\mathrm{C}-2), 81.5$ (C-4), 89.3 (C-1), 121.0 (C-5'), 122.2 (C-8'), 124.9 (C-7'), $126.2\left(\mathrm{C}-6^{\prime}\right), 135.7\left(\mathrm{C}-4^{\prime}\right), 152.8\left(\mathrm{C}-9^{\prime}\right), 162.2\left(\mathrm{C}-2^{\prime}\right), 169.3$, $169.7,169.9,170.4(\mathrm{C}=\mathrm{O})$; HRMS $\left(\mathrm{ESI}^{+}\right) \mathrm{m} / \mathrm{z}$ calcd for $\mathrm{C}_{21} \mathrm{H}_{23} \mathrm{NNaO}_{9} \mathrm{~S}_{2}[\mathrm{M}+\mathrm{Na}]^{+} 520.0712$, found 520.0719. Anal. calcd for $\mathrm{C}_{21} \mathrm{H}_{23} \mathrm{NO}_{9} \mathrm{~S}_{2}$ : C, 50.69; H, 4.66; N, 2.82; S, 12.89 . Found: C, 50.49; H, 4.67; N, 2.85; S, 12.78 .

2-Pyrimidinyl 2,3,5,6-Tetra-O-acetyl-1-thio- $\beta$-D-galactofuranoside (4). This intermediate was synthesized according to the general procedure A from $1(2 \mathrm{~g}, 5.12 \mathrm{mmol})$ at room temperature. Chromatographic purification (light petroleum/ EtOAc, $1: 1)$ gave $4(2.24 \mathrm{~g}, 99 \%)$ as a yellow oil: $R_{f} 0.3$ (light petroleum/EtOAc, 1:1); $[\alpha]^{20}{ }_{\mathrm{D}}-96.9\left(c\right.$ 1.03, $\left.\mathrm{CH}_{2} \mathrm{Cl}_{2}\right) ;{ }^{1} \mathrm{H} \mathrm{NMR}$ $\left(400 \mathrm{MHz}, \mathrm{CDCl}_{3}\right) \delta 2.00,2.10,2.11,2.12\left(4 \mathrm{~s}, 12 \mathrm{H}, \mathrm{CH}_{3} \mathrm{CO}\right)$, 4.16 (dd, $1 \mathrm{H}, J=7.1,11.9 \mathrm{~Hz}, \mathrm{H}-6 \mathrm{a}$ ), 4.31 (dd, $1 \mathrm{H}, J=4.3$, $11.9 \mathrm{~Hz}, \mathrm{H}-6 \mathrm{~b}), 4.39$ (t, $1 \mathrm{H}, J=4.8 \mathrm{~Hz}, \mathrm{H}-4), 5.11(\mathrm{dd}, 1 \mathrm{H}, J$ $=1.0,4.8 \mathrm{~Hz}, \mathrm{H}-3), 5.36(\mathrm{t}, 1 \mathrm{H}, J=1.8 \mathrm{~Hz}, \mathrm{H}-2), 5.40(\mathrm{dt}, 1 \mathrm{H}$, $J=4.8,7.1 \mathrm{~Hz}, \mathrm{H}-5), 6.47(\mathrm{br} \mathrm{s}, 1 \mathrm{H}, \mathrm{H}-1), 7.03(\mathrm{t}, 1 \mathrm{H}, J=4.8$ $\left.\mathrm{Hz}, \mathrm{H}-5^{\prime}\right), 8.54$ (d, $\left.2 \mathrm{H}, J=4.8 \mathrm{~Hz}, \mathrm{H}-4^{\prime}, \mathrm{H}-6{ }^{\prime}\right) ;{ }^{13} \mathrm{C}$ NMR (100 $\left.\mathrm{MHz}, \mathrm{CDCl}_{3}\right) \delta 20.7,20.7,20.8,20.9\left(\mathrm{CH}_{3} \mathrm{CO}\right), 62.6(\mathrm{C}-6), 69.3$ (C-5), 76.7 (C-3), 80.8 (C-2), 81.4 (C-4), 87.5 (C-1), 117.6 $\left(\mathrm{C}-5^{\prime}\right), 157.6\left(\mathrm{C}-4^{\prime}, \mathrm{C}-6^{\prime}\right), 169.5,169.8,170.1,170.5(\mathrm{C}=\mathrm{O}), 169.6$ $\left(\mathrm{C}-2^{\prime}\right)$; HRMS $\left(\mathrm{ESI}^{+}\right) \mathrm{m} / \mathrm{z}$ calcd for $\mathrm{C}_{18} \mathrm{H}_{22} \mathrm{~N}_{2} \mathrm{NaO}_{9} \mathrm{~S}[\mathrm{M}+\mathrm{Na}]^{+}$ 465.0944, found 465.0952. Anal. calcd for $\mathrm{C}_{18} \mathrm{H}_{22} \mathrm{~N}_{2} \mathrm{O}_{9} \mathrm{~S}$ : C, 48.86; H, 5.01; N, 6.33; S, 7.25. Found: C, 48.68; H, 4.94; N, 6.26; S, 7.09.

General Procedure B for the Preparation of Unprotected Thiofuranosyl Donors. To a solution of the peracetylated thioglycofuranoside $(4.2 \mathrm{mmol})$ in anhyd methanol (90 $\mathrm{mL}$ ) was added a $0.1 \mathrm{M}$ solution of sodium methylate in methanol $(4.2 \mathrm{~mL}, 0.42 \mathrm{mmol})$. The mixture was stirred at room temperature until no starting product was detected by TLC. Neutralization was then carefully performed by adding Amberlite IR-120 ( $\mathrm{H}^{+}$-form). The resin was filtered off and the solvent removed under reduced pressure. The desired unprotected glycofuranoside was isolated and then used without further purification.

2-Benzimidazolyl 1-Thio- $\beta$-D-galactofuranoside (5). This compound was synthesized according to the general procedure B from $2(2.22 \mathrm{~g}, 4.62 \mathrm{mmol})$ and isolated as a colorless foam (1.44 g, 100\%): $R_{f} 0.1\left(\mathrm{CH}_{2} \mathrm{Cl}_{2} / \mathrm{MeOH}, 9: 1\right) ;[\alpha]^{20}{ }_{\mathrm{D}}-205.2(c$ $1.02, \mathrm{MeOH}) ;{ }^{1} \mathrm{H}$ NMR $\left(400 \mathrm{MHz}, \mathrm{CD}_{3} \mathrm{OD}\right) \delta 3.62(\mathrm{~d}, 2 \mathrm{H}, J=$ $6.4 \mathrm{~Hz}, \mathrm{H}-6 \mathrm{a}, \mathrm{H}-6 \mathrm{~b}$ ), 3.77 (td, 1H, $J=3.1,6.4 \mathrm{~Hz}, \mathrm{H}-5$ ), 4.11-
4.18 (m 3H, H-2, H-3, H-4), 5.75 (d, 1H, $J=3.9$ Hz, H-1), 7.19$7.23\left(\mathrm{~m}, 2 \mathrm{H}, \mathrm{H}-6^{\prime}, \mathrm{H}-7^{\prime}\right), 7.48-7.52\left(\mathrm{~m}, 2 \mathrm{H}, \mathrm{H}-5^{\prime}, \mathrm{H}-8^{\prime}\right) ;{ }^{13} \mathrm{C}$ NMR (100 MHz, CD $\left.{ }_{3} \mathrm{OD}\right) \delta 64.1(\mathrm{C}-6), 72.1$ (C-5), 78.0 (C-3), 83.4 (C-2), 84.5 (C-4), 92.1 (C-1), 115.3 (C-5', C-8'), 123.6 (C-6', C-7'), $140.5\left(\mathrm{C}-4^{\prime}, \mathrm{C}-9^{\prime}\right), 149.6\left(\mathrm{C}-2^{\prime}\right)$; HRMS $\left(\mathrm{FAB}^{+}\right) \mathrm{m} / \mathrm{z}$ calcd for $\mathrm{C}_{13} \mathrm{H}_{17} \mathrm{~N}_{2} \mathrm{O}_{5} \mathrm{~S}[\mathrm{M}+\mathrm{H}]^{+} 313.0858$, found 313.0855 .

2-Benzothiazolyl 1-Thio-D-galactofuranoside (6). Compound $3(840 \mathrm{mg}, 1.69 \mathrm{mmol})$ was dissolved in a mixture of $\mathrm{MeOH} / \mathrm{water}_{\mathrm{NEt}} \mathrm{NE}_{3}(12 \mathrm{~mL} / 2.5 \mathrm{~mL} / 2.5 \mathrm{~mL})$. After stirring at room temperature for $1 \mathrm{~h}$, the solvent was removed under reduced pressure. The residue was then purified by flash chromatography on silica gel $\left(\mathrm{CH}_{2} \mathrm{Cl}_{2} / \mathrm{MeOH}, 9: 1\right)$ to give an anomeric mixture $(\alpha / \beta, 1: 6.7)$ of $\mathbf{6}(370 \mathrm{mg}, 67 \%)$ as a colorless foam. 6 $\alpha$ : $R_{f} 0.2\left(\mathrm{CH}_{2} \mathrm{Cl}_{2} / \mathrm{EtOAc}, 9: 1\right) ;{ }^{1} \mathrm{H} \mathrm{NMR}(400 \mathrm{MHz}$, $\left.\mathrm{CD}_{3} \mathrm{OD}\right) \delta 3.60-3.68(\mathrm{~m}, 2 \mathrm{H}, \mathrm{H}-6 \mathrm{a}, \mathrm{H}-6 \mathrm{~b}), 3.82-3.85(\mathrm{~m}, 1 \mathrm{H}$, $\mathrm{H}-5$ ), 4.03 (t, $1 \mathrm{H}, J=4.0 \mathrm{~Hz}, \mathrm{H}-4), 4.29(\mathrm{t}, 1 \mathrm{H}, J=4.1 \mathrm{~Hz}$, $\mathrm{H}-3), 4.33(\mathrm{t}, 1 \mathrm{H}, J=4.4 \mathrm{~Hz}, \mathrm{H}-2), 6.23(\mathrm{~d}, 1 \mathrm{H}, J=4.4 \mathrm{~Hz}$, $\mathrm{H}-1$ ), 7.36 (td, $\left.1 \mathrm{H}, J=1.2,8.4 \mathrm{~Hz}, \mathrm{H}-6^{\prime}\right), 7.45$ (td, $1 \mathrm{H}, J=1.5$, $\left.8.4 \mathrm{~Hz}, \mathrm{H}-7^{\prime}\right), 7.84\left(\mathrm{dd}, 1 \mathrm{H}, J=1.2,8.4 \mathrm{~Hz}, \mathrm{H}-8^{\prime}\right), 7.87$ (dd, $\left.1 \mathrm{H}, J=1.5,8.4 \mathrm{~Hz}, \mathrm{H}-5^{\prime}\right) ;{ }^{13} \mathrm{C}$ NMR $\left(100 \mathrm{MHz}, \mathrm{CD}_{3} \mathrm{OD}\right) \delta 64.0$ (C-6), 73.0 (C-5), 78.2 (C-3), 79.4 (C-2), 87.3 (C-4), 91.8 (C-1), $122.4\left(\mathrm{C}-5^{\prime}\right), 122.5\left(\mathrm{C}-8^{\prime}\right), 126.0\left(\mathrm{C}-7^{\prime}\right), 127.5\left(\mathrm{C}-6^{\prime}\right), 136.8$ (C-4'), $154.0\left(\mathrm{C}^{\prime} 9^{\prime}\right), 167.6$ (C-2'). 6 $\beta: R_{f} 0.3\left(\mathrm{CH}_{2} \mathrm{Cl}_{2} / \mathrm{EtOAc}, 9: 1\right)$; ${ }^{1} \mathrm{H}$ NMR $\left(400 \mathrm{MHz}, \mathrm{CD}_{3} \mathrm{OD}\right) \delta 3.63-3.64(\mathrm{~m}, 2 \mathrm{H}, \mathrm{H}-6 \mathrm{a}, \mathrm{H}-6 \mathrm{~b})$, $3.79(\mathrm{td}, 1 \mathrm{H}, J=3.0,6.1 \mathrm{~Hz}, \mathrm{H}-5), 4.15-4.17$ (m, 1H, H-4), $4.19-4.21(\mathrm{~m}, 2 \mathrm{H}, \mathrm{H}-2, \mathrm{H}-3), 6.00(\mathrm{~d}, 1 \mathrm{H}, J=2.6 \mathrm{~Hz}, \mathrm{H}-1)$, $7.36\left(\mathrm{td}, 1 \mathrm{H}, J=1.2,8.4 \mathrm{~Hz}, \mathrm{H}-6^{\prime}\right), 7.45(\mathrm{td}, 1 \mathrm{H}, J=1.5,8.4$ $\left.\mathrm{Hz}, \mathrm{H}-7^{\prime}\right), 7.84\left(\mathrm{dd}, 1 \mathrm{H}, J=1.2,8.4 \mathrm{~Hz}, \mathrm{H}-8^{\prime}\right), 7.87$ (td, $1 \mathrm{H}, J$ $\left.=1.5,8.4 \mathrm{~Hz}, \mathrm{H}-5^{\prime}\right) ;{ }^{13} \mathrm{C}$ NMR $\left(100 \mathrm{MHz}, \mathrm{CD}_{3} \mathrm{OD}\right) \delta 64.2$ (C-6), 72.0 (C-5), 78.1 (C-3), 83.5 (C-2), 85.4 (C-4), 92.9 (C-1), 122.4 (C-5'), 122.5 (C-8'), 126.0 (C-7'), 127.5 (C-6'), 136.8 $\left(\mathrm{C}-4^{\prime}\right), 154.0\left(\mathrm{C}-9^{\prime}\right), 167.5\left(\mathrm{C}-2^{\prime}\right)$; HRMS $\left(\mathrm{ESI}^{+}\right) \mathrm{m} / \mathrm{z}$ calcd for $\mathrm{C}_{13} \mathrm{H}_{16} \mathrm{NO}_{5} \mathrm{~S}_{2}[\mathrm{M}+\mathrm{H}]^{+} 330.0470$, found 330.0478 .

2-Pyrimidinyl 1-Thio- $\beta$-D-galactofuranoside (7). This compound was synthesized as described in general procedure B from $4(2.23 \mathrm{~g}, 5.04 \mathrm{mmol})$ and isolated as a yellow foam (1.35 g, 98\%): $R_{f} 0.4\left(\mathrm{CH}_{2} \mathrm{Cl}_{2} / \mathrm{MeOH}, 4: 1\right) ;[\alpha]^{20}{ }_{\mathrm{D}}-260.9(c 1.01$, $\mathrm{MeOH}) ;{ }^{1} \mathrm{H}$ NMR $\left(400 \mathrm{MHz}, \mathrm{CD}_{3} \mathrm{OD}\right) \delta 3.59(\mathrm{~d}, 2 \mathrm{H}, J=6.9$ $\mathrm{Hz}, \mathrm{H}-6 \mathrm{a}, \mathrm{H}-6 \mathrm{~b}), 3.75$ (td, $1 \mathrm{H}, J=3.1,6.9 \mathrm{~Hz}, \mathrm{H}-5), 4.05$ (dd, $1 \mathrm{H}, J=3.1,6.0 \mathrm{~Hz}, \mathrm{H}-4), 4.16(\mathrm{dd}, 1 \mathrm{H}, J=4.1,6.0 \mathrm{~Hz}, \mathrm{H}-3)$, $4.21(\mathrm{dd}, 1 \mathrm{H}, J=3.8,4.1 \mathrm{~Hz}, \mathrm{H}-2), 6.20(\mathrm{~d}, 1 \mathrm{H}, J=3.8 \mathrm{~Hz}$, $\mathrm{H}-1), 7.18\left(\mathrm{t}, 1 \mathrm{H}, J=5.1 \mathrm{~Hz}, \mathrm{H}-5^{\prime}\right), 8.59(\mathrm{~d}, 2 \mathrm{H}, J=5.1 \mathrm{~Hz}$, $\left.\mathrm{H}-4^{\prime}, \mathrm{H}-6{ }^{\prime}\right) ;{ }^{13} \mathrm{C}$ NMR $\left(100 \mathrm{MHz}, \mathrm{CD}_{3} \mathrm{OD}\right) \delta 64.2(\mathrm{C}-6), 72.3$ (C-5), 78.2 (C-3), 82.9 (C-2), 85.0 (C-4), 90.1 (C-1), 118.7 (C-5'), $158.9\left(\mathrm{C}-4^{\prime}, \mathrm{C}-6^{\prime}\right), 172.2\left(\mathrm{C}-2^{\prime}\right)$; HRMS $\left(\mathrm{ESI}^{+}\right) \mathrm{m} / \mathrm{z}$ calcd for $\mathrm{C}_{10} \mathrm{H}_{14} \mathrm{KN}_{2} \mathrm{O}_{5} \mathrm{~S}[\mathrm{M}+\mathrm{K}]^{+} 313.0260$, found 313.0252 .

General Procedure $\mathrm{C}$ for the Preparation of Ammonium Glycofuranosyl 1-Phosphates. To a solution of the 2-benzimidazolyl glycofuranoside (104 $\mathrm{mg}, 0.34 \mathrm{mmol}$ ) in dry $\mathrm{DMF}(1 \mathrm{~mL})$ was added a solution of orthophosphoric acid (238 $\mathrm{mg}, 2.43 \mathrm{mmol})$ in dry DMF $(1.4 \mathrm{~mL})$. This mixture was then stirred at room temperature for an appropriate period, cooled to $0{ }^{\circ} \mathrm{C}$, and then diluted with water $(15 \mathrm{~mL})$. A saturated solution of aq $\mathrm{Ba}(\mathrm{OH})_{2}$ was added dropwise until the $\mathrm{pH}$ value reached 8-9. The precipitate of $\mathrm{Ba}_{3}\left(\mathrm{PO}_{4}\right)_{2}$ could be removed by centrifugation $\left(15000 \mathrm{rpm}, 0{ }^{\circ} \mathrm{C}, 15 \mathrm{~min}\right)$ and the supernatant liquid filtered. The resulting filtrate and washings (3 $\times 5 \mathrm{~mL}$ ) were combined and carefully neutralized by addition of Amberlite IR-120 ( $\mathrm{H}^{+}$-form). After removal of the resin, the solution was freeze-dried and the residue successively added of absolute EtOH $(2 \mathrm{~mL})$ and EtOAc $(12 \mathrm{~mL})$. The precipitate was collected by centrifugation (15 $000 \mathrm{rpm}, 0{ }^{\circ} \mathrm{C}, 15 \mathrm{~min}$ ), washed in the manner described above, and added to $\mathrm{Et}_{2} \mathrm{O}(14$ $\mathrm{mL}$ ). The solvent was removed after centrifugation (15 000 $\mathrm{rpm}, 0{ }^{\circ} \mathrm{C}, 15 \mathrm{~min}$ ), and the crude barium salt was dried under gentle vaccum. The solid thus obtained was then dissolved in water $(5 \mathrm{~mL})$ and loaded on an Amberlite IR-120 $\left(\mathrm{NH}_{4}{ }^{+}\right.$-form) column $(1 \times 15 \mathrm{~cm})$. After a slow elution with water $(45 \mathrm{~mL})$, the $\mathrm{pH}$ value of the effluent was adjusted at $8.5-9$ by adding few drops of $1 \mathrm{M}$ aq solution of $\mathrm{NH}_{4} \mathrm{OH}$. This effluent was then freeze-dried, and the residue was loaded on an Amberlyst A-26 $\left(\mathrm{CO}_{3}{ }^{2-}\right.$-form $)$ column $(1 \times 15 \mathrm{~cm})$. Neutral compounds were 
first removed by elution with water $(50 \mathrm{~mL})$, and the target phosphate was recovered by elution with $0.21 \mathrm{M}$ aq solution of $\left(\mathrm{NH}_{4}\right)_{2} \mathrm{CO}_{3}(50 \mathrm{~mL})$. Finally, freeze-drying yielded the desired ammonium glycofuranosyl 1-phosphate.

Ammonium D-Galactofuranosyl 1-Phosphate (9). This phosphate was obtained according to the general procedure $\mathrm{C}$ from $\mathbf{5}(108 \mathrm{mg}, 0.35 \mathrm{mmol})$ after stirring at room temperature for $18 \mathrm{~min}$. An anomeric mixture of $\mathbf{9}(\alpha / \beta, 1.2: 1)$ was then isolated as an amorphous and colorless solid $(56 \mathrm{mg}, 58 \%$ ): 9 $\alpha$ : ${ }^{1} \mathrm{H}$ NMR $\left(500 \mathrm{MHz}, \mathrm{D}_{2} \mathrm{O}\right) \delta 3.58(\mathrm{dd}, 1 \mathrm{H}, J=7.1,11.7$ $\mathrm{Hz}, \mathrm{H}-6 \mathrm{a}$ ), 3.65 (dd, $1 \mathrm{H}, J=4.6,11.7 \mathrm{~Hz}, \mathrm{H}-6 \mathrm{~b}$ ), 3.70 (ddd, $1 \mathrm{H}, J=4.6,7.1,9.4 \mathrm{~Hz}, \mathrm{H}-5), 3.76(\mathrm{dd}, 1 \mathrm{H}, J=7.4,9.4 \mathrm{~Hz}$, H-4), 4.05 (dd, $1 \mathrm{H}, J=4.6,8.2 \mathrm{~Hz}, \mathrm{H}-2), 4.19$ (dd, $1 \mathrm{H}, J=$ $7.4,8.2 \mathrm{~Hz}, \mathrm{H}-3), 5.45$ (dd, $1 \mathrm{H}, J=4.6,4.8 \mathrm{~Hz}, \mathrm{H}-1) ;{ }^{13} \mathrm{C}$ NMR (125 MHz, $\left.\mathrm{D}_{2} \mathrm{O}\right) \delta 63.5$ (C-6), $72.9(\mathrm{C}-5), 75.1(\mathrm{C}-3), 77.8$ $(\mathrm{d}, J=7.2 \mathrm{~Hz}, \mathrm{C}-2), 82.3(\mathrm{C}-4), 97.4(\mathrm{~d}, J=5.6 \mathrm{~Hz}, \mathrm{C}-1) ;{ }^{31} \mathrm{P}$ NMR $\left(162 \mathrm{MHz}, \mathrm{D}_{2} \mathrm{O}\right) \delta 2.2 ; 9 \beta:{ }^{1} \mathrm{H},{ }^{13} \mathrm{C}$, and ${ }^{31} \mathrm{P}$ NMR data were identical to the reported data: ${ }^{14 a}$ HRMS $\left(\mathrm{ESI}^{-}\right) \mathrm{m} / \mathrm{z}$ calcd for $\mathrm{C}_{6} \mathrm{H}_{12} \mathrm{O}_{9} \mathrm{P}\left[\mathrm{M}-\mathrm{NH}_{4}-\mathrm{NH}_{3}\right]^{-}$259.0219, found, 259.0213 .

2-Benzimidazolyl 2,3,5,6-Tetra- $O$-acetyl-1-thio- $\beta$-D-glucofuranoside (11). Compound $\mathbf{1 1}$ was prepared as described in general procedure A starting from $10(0.56 \mathrm{~g}, 1.43 \mathrm{mmol})$ under reflux. Chromatographic purification (light petroleum/ EtOAc, 3:2) gave $\mathbf{1 1}(0.46 \mathrm{~g}, 67 \%)$ as a colorless oil: $R_{f} 0.3$ (light petroleum/EtOAc, $1: 1$ ); $[\alpha]^{20}{ }_{\mathrm{D}}-147.0\left(c\right.$ 1.04, $\mathrm{CH}_{2} \mathrm{Cl}_{2}$ ); ${ }^{1} \mathrm{H}$ NMR $\left(400 \mathrm{MHz}, \mathrm{CD}_{3} \mathrm{OD}\right) \delta 1.86,1.95,1.99,2.10(4 \mathrm{~s}, 12 \mathrm{H}$, $\mathrm{CH}_{3} \mathrm{CO}$ ), 4.09 (dd, $1 \mathrm{H}, J=5.1,12.5 \mathrm{~Hz}, \mathrm{H}-6 \mathrm{a}$ ), 4.47 (dd, $1 \mathrm{H}$, $J=3.8,9.1 \mathrm{~Hz}, \mathrm{H}-4), 4.56(\mathrm{dd}, 1 \mathrm{H}, J=2.5,12.5 \mathrm{~Hz}, \mathrm{H}-6 \mathrm{~b})$, $5.26-5.31(\mathrm{~m}, 1 \mathrm{H}, \mathrm{H}-5), 5.31(\mathrm{br} \mathrm{s}, 1 \mathrm{H}, \mathrm{H}-2), 5.42(\mathrm{~d}, 1 \mathrm{H}, J=$ $3.8 \mathrm{~Hz}, \mathrm{H}-3), 5.96$ (s, $1 \mathrm{H}, \mathrm{H}-1), 7.20-7.23$ (m, 2H, H-6', H-7'), $7.52-7.55\left(\mathrm{~m}, 2 \mathrm{H}, \mathrm{H}-5^{\prime}, \mathrm{H}-8^{\prime}\right) ;{ }^{13} \mathrm{C}$ NMR $\left(100 \mathrm{MHz}, \mathrm{CD}_{3} \mathrm{OD}\right) \delta$ 20.5, 20.6, 20.6, $20.8\left(\mathrm{CH}_{3} \mathrm{CO}\right), 64.2$ (C-6), $69.3(\mathrm{C}-5), 75.0$ (C-3), 80.9 (C-4), 82.4 (C-2), 90.3 (C-1), 115.6 (C-5', C-8'), 124.0 (C-6', C-7'), 140.4 (C-4', C-9'), 147.4 (C-2'), 170.4, 170.8, 171.3, $172.4(\mathrm{C}=\mathrm{O})$. Anal. calcd for $\mathrm{C}_{21} \mathrm{H}_{24} \mathrm{~N}_{2} \mathrm{O}_{9} \mathrm{~S}$ : C, 52.49; H, 5.03; N, 5.83; S, 6.67. Found: C, 52.37; H, 5.00; N, 5.67; S, 6.70.

2-Benzimidazolyl 1-Thio- $\beta$-D-glucofuranoside (12). Compound 12 was prepared according to the general procedure B from 11 (410 mg, $0.85 \mathrm{mmol}$ ) and isolated as a colorless foam (266 mg, 100\%): $R_{f} 0.1\left(\mathrm{CH}_{2} \mathrm{Cl}_{2} / \mathrm{MeOH}, 9: 1\right) ;[\alpha]^{20}{ }_{\mathrm{D}}-226.4(c$ 1.04, $\mathrm{MeOH}) ;{ }^{1} \mathrm{H}$ NMR (400 MHz, CD $\left.{ }_{3} \mathrm{OD}\right) \delta 3.65(\mathrm{dd}, 1 \mathrm{H}, J=$ $5.6,11.7 \mathrm{~Hz}, \mathrm{H}-6 \mathrm{a}$ ), 3.79 (dd, $1 \mathrm{H}, J=3.6,11.7 \mathrm{~Hz}, \mathrm{H}-6 \mathrm{~b}$ ), 4.06 (ddd, $1 \mathrm{H}, J=3.6,5.6,8.0 \mathrm{~Hz}, \mathrm{H}-5), 4.17$ (dd, $1 \mathrm{H}, J=3.6,8.0$ $\mathrm{Hz}, \mathrm{H}-4), 4.25$ (dd, $1 \mathrm{H}, J=1.1,3.6 \mathrm{~Hz}, \mathrm{H}-3$ ), 4.37 (br s, $1 \mathrm{H}$, $\mathrm{H}-2), 5.75(\mathrm{~d}, 1 \mathrm{H}, J=1.3 \mathrm{~Hz}, \mathrm{H}-1), 7.16-7.21$ (m, 2H, H-6', $\left.\mathrm{H}-7^{\prime}\right), 7.46-7.51\left(\mathrm{~m}, 2 \mathrm{H}, \mathrm{H}-5^{\prime}, \mathrm{H}-8^{\prime}\right) ;{ }^{13} \mathrm{C}$ NMR $(100 \mathrm{MHz}$, $\left.\mathrm{CD}_{3} \mathrm{OD}\right) \delta 65.1$ (C-6), 71.3 (C-5), 77.2 (C-3), 84.0 (C-2), 84.3 (C-4), 92.3 (C-1), 115.1 (C-5', C-8'), 123.4 (C-6', C-7'), 140.5 (C-4', C-9'), $150.8\left(\mathrm{C}-2^{\prime}\right)$; HRMS $\left(\mathrm{ESI}^{+}\right) \mathrm{m} / \mathrm{z}$ calcd for $\mathrm{C}_{13} \mathrm{H}_{16} \mathrm{~N}_{2} \mathrm{NaO}_{5} \mathrm{~S}[\mathrm{M}+\mathrm{Na}]^{+} 335.0678$, found, 335.0681.

2-Benzimidazolyl 2,3,5,6-Tetra-O-acetyl-1-thio- $\alpha$-D-mannofuranoside (14). Synthesis of 14 was performed according to the general procedure A from $13(0.7 \mathrm{~g}, 1.79 \mathrm{mmol})$ at room temperature. Chromatographic purification (light petroleum/ EtOAc, $1: 1)$ gave $14(0.61 \mathrm{~g}, 71 \%)$ as a white solid: $R_{f} 0.4$ (light petroleum/EtOAc, $1: 1) ; \mathrm{mp} 154^{\circ} \mathrm{C}\left(\mathrm{CH}_{2} \mathrm{Cl}_{2} /\right.$ cyclohexane $) ;[\alpha]^{20}{ }_{\mathrm{D}}$ +83.6 (c 1.01, $\mathrm{CH}_{2} \mathrm{Cl}_{2}$ ); ${ }^{1} \mathrm{H}$ NMR $\left(400 \mathrm{MHz}, \mathrm{CD}_{3} \mathrm{OD}\right) \delta 1.95$, 1.96, 2.02, $2.07\left(4 \mathrm{~s}, 12 \mathrm{H}, \mathrm{CH}_{3} \mathrm{CO}\right), 4.07(\mathrm{dd}, 1 \mathrm{H}, J=5.8,12.3$ $\mathrm{Hz}, \mathrm{H}-6 \mathrm{a}$ ), 4.49 (dd, $1 \mathrm{H}, J=2.3,12.3 \mathrm{~Hz}, \mathrm{H}-6 \mathrm{~b}$ ), 4.54 (dd, $1 \mathrm{H}$, $J=2.8,9.2 \mathrm{~Hz}, \mathrm{H}-4), 5.27$ (ddd, $1 \mathrm{H}, J=2.3,5.8,9.2 \mathrm{~Hz}, \mathrm{H}-5$ ), $5.54-5.58(\mathrm{~m}, 2 \mathrm{H}, \mathrm{H}-2, \mathrm{H}-3), 5.96(\mathrm{~d}, 1 \mathrm{H}, J=6.1 \mathrm{~Hz}, \mathrm{H}-1)$, $7.22-7.26$ (m, 2H, H-6', H-7'), 7.54 (br s, $\left.2 \mathrm{H}, \mathrm{H}-5^{\prime}, \mathrm{H}-8^{\prime}\right) ;{ }^{13} \mathrm{C}$ NMR (100 MHz, CD $\mathrm{OD}) \delta 20.2,20.4,20.5,20.7\left(\mathrm{CH}_{3} \mathrm{CO}\right), 63.8$ (C-6), 69.1 (C-5), 71.8 (C-3), 76.8 (C-2), 78.7 (C-4), 88.3 (C-1), 124.0 (C-6', C-7'), 147.0 (C-2'), 171.2, 171.3, 171.8, 172.4 $(\mathrm{C}=\mathrm{O})$. Anal. calcd for $\mathrm{C}_{21} \mathrm{H}_{24} \mathrm{~N}_{2} \mathrm{O}_{9} \mathrm{~S}: \mathrm{C}, 52.49 ; \mathrm{H}, 5.03 ; \mathrm{N}, 5.83$; S, 6.67. Found: C, 52.29; H, 4.96; N, 5.67; S, 6.54.

2-Benzimidazolyl 1-Thio- $\alpha$-D-mannofuranoside (15). This compound was synthesized according to the general procedure B starting from $14(585 \mathrm{mg}, 1.22 \mathrm{mmol})$. After purification, $\mathbf{1 5}$ was obtained as a colorless foam $(380 \mathrm{mg})$ in a quantitative yield: $R_{f} 0.2\left(\mathrm{CH}_{2} \mathrm{Cl}_{2} / \mathrm{MeOH}, 9: 1\right) ;[\alpha]^{20} \mathrm{D}+234.7$ (c 1.00, MeOH); ${ }^{1} \mathrm{H}$ NMR $\left(400 \mathrm{MHz}, \mathrm{CD}_{3} \mathrm{OD}\right) \delta 3.61(\mathrm{dd}, 1 \mathrm{H}, J$ $=5.3,11.7 \mathrm{~Hz}, \mathrm{H}-6 \mathrm{a}), 3.74$ (dd, $1 \mathrm{H}, J=3.1,11.7 \mathrm{~Hz}, \mathrm{H}-6 \mathrm{~b})$, 3.97 (ddd, $1 \mathrm{H}, J=3.1,5.3,8.1 \mathrm{~Hz}, \mathrm{H}-5), 4.07$ (dd, $1 \mathrm{H}, J=2.0$, $8.1 \mathrm{~Hz}, \mathrm{H}-4), 4.22-4.26$ (m, $2 \mathrm{H}, \mathrm{H}-2, \mathrm{H}-3), 5.62-5.65(\mathrm{~m}, 1 \mathrm{H}$, $\mathrm{H}-1), 7.19-7.24$ (m, 2H, H-6', H-7'), 7.49-7.53 (m, 2H, H-5', $\left.\mathrm{H}-8^{\prime}\right) ;{ }^{13} \mathrm{C}$ NMR $\left(100 \mathrm{MHz}, \mathrm{CD}_{3} \mathrm{OD}\right) \delta 64.6$ (C-6), 70.7 (C-5), 72.4 (C-3), 78.9 (C-2), 81.9 (C-4), 91.2 (C-1), 115.4 (C-5', C-8'), $123.7\left(\mathrm{C}-6^{\prime}, \mathrm{C}-7^{\prime}\right), 140.5\left(\mathrm{C}-4^{\prime}, \mathrm{C}-9^{\prime}\right), 148.8\left(\mathrm{C}-2^{\prime}\right)$; HRMS $\left(\mathrm{ESI}^{+}\right)$ $m / z$ calcd for $\mathrm{C}_{13} \mathrm{H}_{16} \mathrm{~N}_{2} \mathrm{NaO}_{5} \mathrm{~S}[\mathrm{M}+\mathrm{Na}]^{+} 335.0678$, found, 335.0673 .

It is interesting to note that $\mathrm{H}-1$ is characterized by a complex and unusual signal assigned by ${ }^{1} \mathrm{H}$ NMR. Indeed, because signals from H-2 and H-3 are superimposed, secondorder effects arose out of virtual long-range coupling and so resulted in producing a more complex signal (six peaks) than the expected doublet. See for example: Kotowycz, G.; Lemieux, R. U. Chem. Rev. 1973, 73, 669-698.

n-Octyl 6-O-Triphenylmethyl- $\beta$-D-galactofuranoside (17). To a suspension of 16 (20 g, $68.41 \mathrm{mmol})$ in dry dichloromethane $(200 \mathrm{~mL}$ ) was added $4-N, N$-(dimethylamino)$1-N$-triphenylmethylpyridinium chloride (32.91 g, $205.24 \mathrm{mmol})$. The mixture was heated under reflux for $24 \mathrm{~h}$, cooled to room temperature, concentrated, and then diluted with ethyl acetate $(200 \mathrm{~mL})$. The solution was washed successively with $5 \%$ aq $\mathrm{HCl}(30 \mathrm{~mL})$, a saturated solution of aq $\mathrm{NaHCO}_{3}(30 \mathrm{~mL})$, and brine $(30 \mathrm{~mL})$. The aqueous layers thus obtained were extracted with ethyl acetate $(3 \times 30 \mathrm{~mL})$ and the combined organic layers dried $\left(\mathrm{MgSO}_{4}\right)$ and concentrated. Purification by flash chromatography (light petroleum/EtOAc, 3:7) afforded $17(27.42 \mathrm{~g}, 80 \%)$ as a colorless oil: ${ }^{1} \mathrm{H}$ and ${ }^{13} \mathrm{C}$ NMR data of 17 were identical to the reported data. ${ }^{31}$

$\boldsymbol{n}$-Octyl 2,3,5-Tri-O-benzyl-6-O-tosyl- $\boldsymbol{\beta}$-D-galactofuranoside (20). Triethylamine (16.4 $\mathrm{mL}, 116.39 \mathrm{mmol})$, DMAP $(0.28 \mathrm{~g}, 2.33 \mathrm{mmol})$, and tosyl chloride $(6.66 \mathrm{~g}, 34.92 \mathrm{mmol})$ were successively added to a solution of $\mathbf{1 9}$ (13.10 g, 23.28 $\mathrm{mmol})$ in dry dichloromethane $(130 \mathrm{~mL})$. The mixture was heated under reflux for $8 \mathrm{~h}$, then cooled to room temperature, diluted with dichloromethane $(70 \mathrm{~mL})$, and washed successively with $5 \%$ aq $\mathrm{HCl}(50 \mathrm{~mL})$, a saturated solution of aq $\mathrm{NaHCO}_{3}(50 \mathrm{~mL})$, and water $(50 \mathrm{~mL})$. The aqueous layers were extracted twice with dichloromethane $(2 \times 50 \mathrm{~mL})$ and the combined organic layers dried $\left(\mathrm{MgSO}_{4}\right)$ and concentrated. The residue was finally purified by flash chromatography on silica gel (light petroleum/EtOAc, 9:1) to give 20 (16.09 g, 96\%) as a colorless oil: $R_{f} 0.3$ (light petroleum/EtOAc, $4: 1$ ); $[\alpha]^{20}{ }_{\mathrm{D}}-46.1$ (c $\left.1.02, \mathrm{CH}_{2} \mathrm{Cl}_{2}\right) ;{ }^{1} \mathrm{H}$ NMR $\left(400 \mathrm{MHz}, \mathrm{CDCl}_{3}\right) \delta 0.88(\mathrm{t}, 3 \mathrm{H}, J=$ $\left.6.8 \mathrm{~Hz}, \mathrm{CH}_{3}\right), 1.22-1.35\left[\mathrm{~m}, 10 \mathrm{H},\left(\mathrm{CH}_{2}\right)_{5}\right], 1.50-1.56(\mathrm{~m}, 2 \mathrm{H}$, $\left.\mathrm{OCH}_{2} \mathrm{CH}_{2}\right), 2.41\left(\mathrm{~s}, 3 \mathrm{H}, \mathrm{H}_{3} \mathrm{CC}_{6} \mathrm{H}_{4} \mathrm{SO}_{2}\right), 3.31(\mathrm{dt}, 1 \mathrm{H}, J=6.8$, $9.7 \mathrm{~Hz}, \mathrm{OCH}_{2} \mathrm{CH}_{2}$ ), $3.58\left(\mathrm{dt}, 1 \mathrm{H}, J=6.9,9.7 \mathrm{~Hz}, \mathrm{OCH}_{2} \mathrm{CH}_{2}\right.$ ), $3.77-3.81$ (m, 1H, H-5), 3.90 (dd, $1 \mathrm{H}, J=3.3,7.1 \mathrm{~Hz}, \mathrm{H}-3$ ), $3.94(\mathrm{dd}, 1 \mathrm{H}, J=1.3,3.3 \mathrm{~Hz}, \mathrm{H}-2), 4.01(\mathrm{dd}, 1 \mathrm{H}, J=3.3,7.1$ $\mathrm{Hz}, \mathrm{H}-4$ ), 4.13 (dd, $1 \mathrm{H}, J=7.4,10.4 \mathrm{~Hz}, \mathrm{H}-6 \mathrm{a}), 4.20$ (dd, $1 \mathrm{H}$, $J=4.3,10.4 \mathrm{~Hz}, \mathrm{H}-6 \mathrm{~b}), 4.25-4.60\left(\mathrm{~m}, 6 \mathrm{H}, \mathrm{OCH}_{2} \mathrm{C}_{6} \mathrm{H}_{5}\right), 4.98$ (br s, 1H, H-1), 7.18-7.37 (m, 17H, $\left.\mathrm{C}_{6} \mathrm{H}_{4}, \mathrm{C}_{6} \mathrm{H}_{5}\right), 7.73(\mathrm{~d}, 2 \mathrm{H}$, $\left.J=8.4 \mathrm{~Hz}, \mathrm{C}_{6} \mathrm{H}_{4}\right) ;{ }^{13} \mathrm{C} \mathrm{NMR}\left(100 \mathrm{MHz} \mathrm{CDCl}_{3}\right) \delta 14.2\left(\mathrm{CH}_{3}\right)$, $21.7\left(\mathrm{H}_{3} \mathrm{CC}_{6} \mathrm{H}_{4} \mathrm{SO}_{2}\right), 22.8,26.2,29.4,29.5,29.5,31.9\left[\left(\mathrm{CH}_{2}\right)_{6}\right]$, $67.9\left(\mathrm{OCH}_{2} \mathrm{CH}_{2}\right), 70.3(\mathrm{C}-6), 72.0,72.1,73.8\left(\mathrm{OCH}_{2} \mathrm{C}_{6} \mathrm{H}_{5}\right), 75.3$ (C-5), 80.2 (C-4), 82.5 (C-3), 88.2 (C-2), 106.0 (C-1), 127.9, 127.9, $128.0,128.0,128.1,128.4,128.4,128.5,128.5,129.9\left(\mathrm{C}_{6} \mathrm{H}_{5}\right.$, $\left.\mathrm{C}_{6} \mathrm{H}_{4}\right), 132.8\left(\mathrm{C}_{\mathrm{p}} \mathrm{C}_{6} \mathrm{H}_{4} \mathrm{SO}_{2}\right), 137.6,137.7,137.7\left(\mathrm{C}_{\text {ipso }} \mathrm{C}_{6} \mathrm{H}_{5}\right)$, $144.9\left(\mathrm{C}_{\text {ipso }} \mathrm{C}_{6} \mathrm{H}_{4} \mathrm{SO}_{2}\right)$. Anal. calcd for $\mathrm{C}_{42} \mathrm{H}_{52} \mathrm{O}_{8} \mathrm{~S}: \mathrm{C}, 70.36 ; \mathrm{H}$, 7.31; S, 4.47. Found: C, 70.08; H, 7.33; S, 4.50.

$\boldsymbol{n}$-Octyl 2,3,5-Tri-O-benzyl- $\boldsymbol{\beta}$-D-fucofuranoside (21). To a solution of 20 (14.95 g, $20.85 \mathrm{mmol})$ in anhyd diethyl ether $(150 \mathrm{~mL})$ was added portionwise LAH $(2.37 \mathrm{~g}, 62.56 \mathrm{mmol})$. After stirring under reflux for $3 \mathrm{~h}$, the mixture was cooled to $0{ }^{\circ} \mathrm{C}$ and carefully added to ethyl acetate $(50 \mathrm{~mL})$. Ammonium fluoride $(20 \mathrm{~g}, 539.96 \mathrm{mmol})$ and then water $(150 \mathrm{~mL})$ were added. The mixture was stirred at room temperature for 10 min, filtered, and the residue washed with diethyl ether (400 $\mathrm{mL}$ ). After separation, the organic layer was successively 
washed with $5 \%$ aq $\mathrm{HCl}(40 \mathrm{~mL})$, a saturated solution of aq $\mathrm{NaHCO}_{3}(40 \mathrm{~mL})$, and brine $(40 \mathrm{~mL})$. The aqueous layers were extracted with diethyl ether $(2 \times 40 \mathrm{~mL})$ and the combined organic layers dried $\left(\mathrm{MgSO}_{4}\right)$ and concentrated. A flash chromatography (light petroleum/EtOAc, 19:1) purification afforded $21(10.29 \mathrm{~g}, 90 \%)$ as a colorless oil: $R_{f} 0.3$ (light petroleum/ EtOAc, 19:1); $[\alpha]{ }^{20}{ }_{\mathrm{D}}-52.7$ (c 1.06, $\left.\mathrm{CH}_{2} \mathrm{Cl}_{2}\right) ;{ }^{1} \mathrm{H}$ NMR (400 MHz, $\left.\mathrm{CDCl}_{3}\right) \delta 0.91\left(\mathrm{t}, 3 \mathrm{H}, J=7.1 \mathrm{~Hz}, \mathrm{CH}_{3}\right), 1.27(\mathrm{~d}, 3 \mathrm{H}, J=6.4$ $\mathrm{Hz}, \mathrm{H}-6), 1.30-1.37\left[\mathrm{~m}, 10 \mathrm{H},\left(\mathrm{CH}_{2}\right)_{5}\right], 1.59-1.66(\mathrm{~m}, 2 \mathrm{H}$, $\left.\mathrm{OCH}_{2} \mathrm{CH}_{2}\right), 3.45\left(\mathrm{dt}, 1 \mathrm{H}, J=6.6,9.6 \mathrm{~Hz}, \mathrm{OCH}_{2} \mathrm{CH}_{2}\right), 3.69-$ 3.76 (m, $\left.2 \mathrm{H}, \mathrm{OCH}_{2} \mathrm{CH}_{2}, \mathrm{H}-5\right), 3.99-4.05$ (m, 3H, H-2, H-3, H-4), 4.40-4.68 (m, 6H, OCH $\left.\mathrm{C}_{6} \mathrm{H}_{5}\right), 5.09(\mathrm{~s}, 1 \mathrm{H}, \mathrm{H}-1), 7.25-7.39$ $\left(\mathrm{m}, 15 \mathrm{H}, \mathrm{C}_{6} \mathrm{H}_{5}\right) ;{ }^{13} \mathrm{C} \mathrm{NMR}\left(100 \mathrm{MHz}, \mathrm{CDCl}_{3}\right) \delta 14.2\left(\mathrm{CH}_{3}\right)$, 16.1 (C-6), 22.8, 26.3, 29.4, 29.5, 29.6, 31.9 [( $\left.\left.\mathrm{CH}_{2}\right)_{6}\right], 67.7$ $\left(\mathrm{OCH}_{2} \mathrm{CH}_{2}\right), 71.3,71.9,72.1\left(\mathrm{OCH}_{2} \mathrm{C}_{6} \mathrm{H}_{5}\right), 73.3(\mathrm{C}-5), 83.3$ (C-3), 83.7 (C-4), 88.7 (C-2), 105.8 (C-1), 127.6, 127.8, 127.9, 128.0, 128.3, 128.4, $128.5\left(\mathrm{C}_{6} \mathrm{H}_{5}\right), 137.8,138.0,138.7\left(\mathrm{C}_{\mathrm{ipso}}\right.$ $\mathrm{C}_{6} \mathrm{H}_{5}$ ). Anal. calcd for $\mathrm{C}_{35} \mathrm{H}_{46} \mathrm{O}_{5}$ : C, 76.89; $\mathrm{H}, 8.48$. Found: $\mathrm{C}$, $76.50 ; \mathrm{H}, 8.44$.

$\boldsymbol{n}$-Octyl $\boldsymbol{\beta}$-D-Fucofuranoside (22). Acetic acid (5 mL) and $\mathrm{Pd}(\mathrm{OAc})_{2}(100 \mathrm{mg})$ were added to a solution of $\mathbf{2 1}(1.03 \mathrm{~g}, 1.88$ $\mathrm{mmol})$ in ethyl acetate $(5 \mathrm{~mL})$. After stirring under hydrogen (1 atm) at room temperature for $72 \mathrm{~h}$, the mixture was concentrated under reduced pressure, and traces of acetic acid were coevaporated with methanol $(5 \times 20 \mathrm{~mL})$. The crude product was chromatographed on silica gel $\left(\mathrm{CH}_{2} \mathrm{Cl}_{2} / \mathrm{MeOH}, 9: 1\right)$ to afford target $22(0.52 \mathrm{~g}, 100 \%)$ as a colorless oil: $R_{f} 0.5$ $\left(\mathrm{CH}_{2} \mathrm{Cl}_{2} / \mathrm{MeOH}, 9: 1\right) ;[\alpha]^{20} \mathrm{D}-89.3$ (c 1.07, $\left.\mathrm{MeOH}\right) ;{ }^{1} \mathrm{H}$ NMR $\left(400 \mathrm{MHz}, \mathrm{CDCl}_{3}+\mathrm{D}_{2} \mathrm{O}\right) \delta 0.87\left(\mathrm{t}, 3 \mathrm{H}, J=6.8 \mathrm{~Hz}, \mathrm{CH}_{3}\right)$, $1.24-1.30\left[\mathrm{~m}, 10 \mathrm{H},\left(\mathrm{CH}_{2}\right)_{5}\right], 1.32(\mathrm{~d}, 3 \mathrm{H}, J=6.6 \mathrm{~Hz}, \mathrm{H}-6)$, $1.52-1.60\left(\mathrm{~m}, 2 \mathrm{H}, \mathrm{OCH}_{2} \mathrm{CH}_{2}\right), 3.43(\mathrm{dt}, 1 \mathrm{H}, J=6.7,9.6 \mathrm{~Hz}$, $\left.\mathrm{OCH}_{2} \mathrm{CH}_{2}\right), 3.72\left(\mathrm{dt}, 1 \mathrm{H}, J=6.9,9.6 \mathrm{~Hz}, \mathrm{OCH}_{2} \mathrm{CH}_{2}\right), 3.90(\mathrm{dd}$, $1 \mathrm{H}, J=2.0,2.3 \mathrm{~Hz}, \mathrm{H}-4$ ), 3.92 (br s, $1 \mathrm{H}, \mathrm{H}-3$ ), 3.97 (br s, $1 \mathrm{H}$, $\mathrm{H}-2$ ), 4.01 (qd, $1 \mathrm{H}, J=2.0,6.6 \mathrm{~Hz}, \mathrm{H}-5$ ), 4.99 (s, 1H, H-1); ${ }^{13} \mathrm{C}$ NMR $\left(100 \mathrm{MHz}, \mathrm{CDCl}_{3}+\mathrm{D}_{2} \mathrm{O}\right) \delta 14.2\left(\mathrm{CH}_{3}\right), 20.1(\mathrm{C}-6)$, $22.7,26.2,29.3,29.4,29.6,31.9\left[\left(\mathrm{CH}_{2}\right)_{6}\right], 67.5(\mathrm{C}-5), 67.8$ $\left(\mathrm{OCH}_{2} \mathrm{CH}_{2}\right), 78.7$ (C-3), $78.8(\mathrm{C}-2), 89.9(\mathrm{C}-4), 107.9$ (C-1). Anal. calcd for $\mathrm{C}_{14} \mathrm{H}_{28} \mathrm{O}_{5}$ : C, 60.84; $\mathrm{H}, 10.21$. Found: C, 60.48; $\mathrm{H}$, 10.03 .

$\boldsymbol{n}$-Octyl 2,3,5-Tri-O-acetyl- $\boldsymbol{\beta}$-D-fucofuranoside (23). To a solution of $22(0.52 \mathrm{~g}, 1.88 \mathrm{mmol})$ in dry pyridine $(4.57 \mathrm{~mL}$, $55.45 \mathrm{mmol})$ was added $\mathrm{Ac}_{2} \mathrm{O}(5.3 \mathrm{~mL}, 55.45 \mathrm{mmol})$. After stirring at room temperature for $21 \mathrm{~h}$, the mixture was evaporated to dryness under reduced pressure, and the resulting oil was then partitioned between ethyl acetate $(100 \mathrm{~mL})$ and $5 \%$ aq $\mathrm{HCl}(20 \mathrm{~mL})$. The organic layer was washed with a saturated solution of aq $\mathrm{NaHCO}_{3}(3 \times 20 \mathrm{~mL})$ and water $(20 \mathrm{~mL})$. The aqueous layers were extracted with ethyl acetate $(2 \times 20 \mathrm{~mL})$ and the combined organic layers dried $\left(\mathrm{MgSO}_{4}\right)$ and concentrated. Purification of the residue by flash chromatography on silica gel (light petroleum/EtOAc, 4:1) gave $\mathbf{2 3}$ $(0.73 \mathrm{~g}, 96 \%)$ as a colorless oil: $R_{f} 0.6$ (light petroleum/EtOAc, 3:2); $[\alpha]^{20}{ }_{\mathrm{D}}-62.4\left(c \mathrm{1.01}, \mathrm{CH}_{2} \mathrm{Cl}_{2}\right) ;{ }^{1} \mathrm{H} \mathrm{NMR}\left(400 \mathrm{MHz}, \mathrm{CDCl}_{3}\right)$ $\delta 0.88\left(\mathrm{t}, 3 \mathrm{H}, J=7.1 \mathrm{~Hz}, \mathrm{CH}_{3}\right), 1.27-1.29\left[\mathrm{~m}, 10 \mathrm{H},\left(\mathrm{CH}_{2}\right)_{5}\right]$, 1.31 (d, $3 \mathrm{H}, J=6.6 \mathrm{~Hz}, \mathrm{H}-6), 1.55-1.63\left(\mathrm{~m}, 2 \mathrm{H}, \mathrm{OCH}_{2} \mathrm{CH}_{2}\right.$ ), $2.08,2.10,2.10\left(3 \mathrm{~s}, 9 \mathrm{H}, \mathrm{CH}_{3} \mathrm{CO}\right), 3.45(\mathrm{dt}, 1 \mathrm{H}, J=6.3,9.7 \mathrm{~Hz}$, $\mathrm{OCH}_{2} \mathrm{CH}_{2}$ ), 3.66 (dt, $1 \mathrm{H}, J=6.9,9.7 \mathrm{~Hz}, \mathrm{OCH}_{2} \mathrm{CH}_{2}$ ), 4.07 (dd, $1 \mathrm{H}, J=4.1,5.9 \mathrm{~Hz}, \mathrm{H}-4), 4.99(\mathrm{dd}, 1 \mathrm{H}, J=1.8,5.9 \mathrm{~Hz}, \mathrm{H}-3)$, 5.00 (br s, $1 \mathrm{H}, \mathrm{H}-1$ ), 5.03 (d, $1 \mathrm{H}, J=1.8 \mathrm{~Hz}, \mathrm{H}-2$ ), 5.15 (qd, $1 \mathrm{H}, J=4.1,6.6 \mathrm{~Hz}, \mathrm{H}-5) ;{ }^{13} \mathrm{C}$ NMR $\left(100 \mathrm{MHz} \mathrm{CDCl}_{3}\right) \delta 14.2$ $\left(\mathrm{CH}_{3}\right), 16.3$ (C-6), 20.8, 20.9, $21.2\left(\mathrm{CH}_{3} \mathrm{CO}\right), 22.7,26.1$, 29.3, $29.4,29.5,31.9\left[\left(\mathrm{CH}_{2}\right)_{6}\right], 67.8\left(\mathrm{OCH}_{2} \mathrm{CH}_{2}\right), 68.8(\mathrm{C}-5), 77.3$ (C-3), 81.9 (C-2), 82.7 (C-4), 105.4 (C-1), 169.9, 170.2, 170.4 $(\mathrm{C}=\mathrm{O})$. Anal. calcd for $\mathrm{C}_{20} \mathrm{H}_{34} \mathrm{O}_{8}$ : C, 59.68; $\mathrm{H}, 8.51$. Found: $\mathrm{C}$, 59.64; H, 8.39.

1,2,3,5-Tetra- $O$-acetyl-D-fucofuranose (24). Tri- $O$-acetylated D-fucofuranoside $23(0.71 \mathrm{~g}, 1.76 \mathrm{mmol})$ was dissolved in dry dichloromethane $(7 \mathrm{~mL})$. Acetic anhydride $(670 \mu \mathrm{L}, 7.13$ $\mathrm{mmol})$ and concd sulfuric acid (20 $\mu \mathrm{L}, 0.38 \mathrm{mmol})$ were successively added. After stirring for $24 \mathrm{~h}$ at room temperature, the reaction was quenched by adding few drops of triethylamine and then concentrated. The residue was finally purified by flash chromatography (light petroleum/EtOAc, 3:2) to give an anomeric mixture $(\alpha / \beta, 1: 4.3)$ of $24(0.55 \mathrm{~g}, 94 \%)$ as a colorless oil: $R_{f} 0.3$ (light petroleum/EtOAc, 3:2). $24 \alpha$ : ${ }^{1} \mathrm{H}$ $\operatorname{NMR}\left(400 \mathrm{MHz}, \mathrm{CDCl}_{3}\right) \delta 1.21(\mathrm{~d}, 3 \mathrm{H}, J=6.4 \mathrm{~Hz}, \mathrm{H}-6), 2.07$, 2.08, 2.09, $2.12\left(4 \mathrm{~s}, 12 \mathrm{H}, \mathrm{CH}_{3} \mathrm{CO}\right), 3.97(\mathrm{t}, 1 \mathrm{H}, J=6.6 \mathrm{~Hz}$, $\mathrm{H}-4), 5.07-5.12(\mathrm{~m}, 1 \mathrm{H}, \mathrm{H}-5), 5.30(\mathrm{dd}, 1 \mathrm{H}, J=4.6,6.6 \mathrm{~Hz}$, $\mathrm{H}-2), 5.50(\mathrm{t}, 1 \mathrm{H}, J=6.6 \mathrm{~Hz}, \mathrm{H}-3), 6.30(\mathrm{~d}, 1 \mathrm{H}, J=4.6 \mathrm{~Hz}$, $\mathrm{H}-1) ;{ }^{13} \mathrm{C} \mathrm{NMR}\left(100 \mathrm{MHz}, \mathrm{CDCl}_{3}\right) \delta 15.8(\mathrm{C}-6), 20.6,20.8,20.8$, $21.2\left(\mathrm{CH}_{3} \mathrm{CO}\right), 69.9(\mathrm{C}-5), 73.7(\mathrm{C}-3), 75.6(\mathrm{C}-2), 82.3(\mathrm{C}-4), 93.2$ $(\mathrm{C}-1), 169.5,170.0,170.0,170.2(\mathrm{C}=\mathrm{O}) . \mathbf{2 4} \beta:{ }^{1} \mathrm{H}$ NMR $(400$ $\left.\mathrm{MHz} \mathrm{CDCl}_{3}\right) \delta 1.30(\mathrm{~d}, 3 \mathrm{H}, J=6.3 \mathrm{~Hz}, \mathrm{H}-6), 2.08,2.11,2.12$, $2.12\left(4 \mathrm{~s}, 12 \mathrm{H}, \mathrm{CH}_{3} \mathrm{CO}\right), 4.21(\mathrm{dd}, 1 \mathrm{H}, J=4.6,5.1 \mathrm{~Hz}, \mathrm{H}-4)$, 5.08 (dd, $1 \mathrm{H}, J=1.8,5.1 \mathrm{~Hz}, \mathrm{H}-3$ ), 5.15 (qd, $1 \mathrm{H}, J=4,6,6.3$ $\mathrm{Hz}, \mathrm{H}-5$ ), 5.17 (dd, $1 \mathrm{H}, J=1.0,1.8 \mathrm{~Hz}, \mathrm{H}-2$ ), 6.18 (br s, $1 \mathrm{H}$, $\mathrm{H}-1) ;{ }^{13} \mathrm{C} \mathrm{NMR}\left(100 \mathrm{MHz}, \mathrm{CDCl}_{3}\right) \delta 16.0$ (C-6), 20.8, 20.8, 21.1, $21.2\left(\mathrm{CH}_{3} \mathrm{CO}\right), 68.6$ (C-5), $75.6(\mathrm{C}-3), 81.0(\mathrm{C}-2), 84.9(\mathrm{C}-4), 99.2$ $(\mathrm{C}-1), 169.3,169.5,169.9,170.2(\mathrm{C}=\mathrm{O})$. Anal. calcd for $\mathrm{C}_{14} \mathrm{H}_{20} \mathrm{O}_{9}$ : C, 50.60; H, 6.07. Found: C, 50.49; H, 6.01.

2-Benzimidazolyl 2,3,5,6-Tetra- $O$-acetyl-1-thio- $\beta$-D-fucofuranoside (25). This compound was synthesized according to the general procedure A from $24(177 \mathrm{mg}, 0.53 \mathrm{mmol})$ at room temperature. Chromatographic purification (light petroleum/EtOAc, 1:1) gave the desired product $25(202 \mathrm{mg}, 90 \%)$ as a colorless foam: $R_{f} 0.3$ (light petroleum/EtOAc, $1: 1$ ); $[\alpha]^{20}{ }_{\mathrm{D}}$ -128.6 (c 1.02, $\mathrm{CH}_{2} \mathrm{Cl}_{2}$ ); ${ }^{1} \mathrm{H} \mathrm{NMR}\left(400 \mathrm{MHz}, \mathrm{CDCl}_{3}\right) \delta 1.34(\mathrm{~d}$, $3 \mathrm{H}, J=6.6 \mathrm{~Hz}, \mathrm{H}-6), 2.10,2.10,2.14\left(3 \mathrm{~s}, 9 \mathrm{H}, \mathrm{CH}_{3} \mathrm{CO}\right), 4.38$ (t, $1 \mathrm{H}, J=5.1 \mathrm{~Hz}, \mathrm{H}-4), 5.16$ (dd, $1 \mathrm{H}, J=2.0,5.2 \mathrm{~Hz}, \mathrm{H}-3$ ), $5.21-5.27(\mathrm{~m}, 1 \mathrm{H}, \mathrm{H}-5), 5.30(\mathrm{t}, 1 \mathrm{H}, J=2.0 \mathrm{~Hz}, \mathrm{H}-2), 5.97(\mathrm{~d}$, $1 \mathrm{H}, J=2.3 \mathrm{~Hz}, \mathrm{H}-1), 7.22-7.26\left(\mathrm{~m}, 2 \mathrm{H}, \mathrm{H}-6^{\prime}, \mathrm{H}-7^{\prime}\right), 7.40-$ $7.70\left(\mathrm{~m}, 2 \mathrm{H}, \mathrm{H}-5^{\prime}, \mathrm{H}-8^{\prime}\right), 9.94(\mathrm{br} \mathrm{s}, 1 \mathrm{H}, \mathrm{NH}) ;{ }^{13} \mathrm{C}$ NMR $(100$ $\left.\mathrm{MHz} \mathrm{CDCl}_{3}\right) \delta 16.1$ (C-6), 20.7, 20.7, $21.2\left(\mathrm{CH}_{3} \mathrm{CO}\right), 68.6$ (C-5), 76.6 (C-3), 81.5 (C-2), 84.4 (C-4), 88.5 (C-1), 110.4, 119.1 (C-5', C-8'), 123.0 (C-6', C-7'), 146.1 (C-2', C-4', C-9'), $169.9,169.9,170.5(\mathrm{C}=\mathrm{O})$; HRMS $\left(\mathrm{ESI}^{+}\right) \mathrm{m} / \mathrm{z}$ calcd for $\mathrm{C}_{19} \mathrm{H}_{22} \mathrm{~N}_{2} \mathrm{NaO}_{7} \mathrm{~S}[\mathrm{M}+\mathrm{Na}]^{+} 445.1045$, found, 445.1045. Anal. calcd for $\mathrm{C}_{19} \mathrm{H}_{22} \mathrm{~N}_{2} \mathrm{O}_{7} \mathrm{~S}$ : C, 54.02; H, 5.25; N, 6.63; S, 7.59. Found: C, 54.01; H, 5.26; N, 6.67; S, 7.77.

2-Benzimidazolyl 1-Thio- $\boldsymbol{\beta}$-D-fucofuranoside (26). Starting from 25 (302 $\mathrm{mg}, 0.71 \mathrm{mmol})$, and according to the general procedure B, the target thiofuranoside $\mathbf{2 6}$ was obtained as a colorless foam $(196 \mathrm{mg})$ in $93 \%$ yield: $R_{f} 0.5\left(\mathrm{CH}_{2} \mathrm{Cl}_{2} /\right.$ $\mathrm{MeOH}, 4: 1) ;[\alpha]^{20}{ }_{\mathrm{D}}-284.0$ (c 1.08, $\left.\mathrm{MeOH}\right) ;{ }^{1} \mathrm{H}$ NMR $(400 \mathrm{MHz}$, $\left.\mathrm{CD}_{3} \mathrm{OD}\right) \delta 1.24(\mathrm{~d}, 3 \mathrm{H}, J=6.4 \mathrm{~Hz}, \mathrm{H}-6), 3.84-3.89(\mathrm{~m}, 2 \mathrm{H}$, $\mathrm{H}-4, \mathrm{H}-5$ ), 3.98 (dd, $1 \mathrm{H}, J=4.0,6.1 \mathrm{~Hz}, \mathrm{H}-3$ ), 4.14 (t, $1 \mathrm{H}, J=$ $4.0 \mathrm{~Hz}, \mathrm{H}-2), 5.77(\mathrm{~d}, 1 \mathrm{H}, J=4.0 \mathrm{~Hz}, \mathrm{H}-1), 7.20-7.24(\mathrm{~m}, 2 \mathrm{H}$, H-6', H-7'), 7.48-7.52 (m, 2H, H-5', H-8'); ${ }^{13} \mathrm{C}$ NMR (100 MHz, $\left.\mathrm{CD}_{3} \mathrm{OD}\right) \delta 19.5(\mathrm{C}-6), 68.5(\mathrm{C}-5), 78.8(\mathrm{C}-3), 83.9(\mathrm{C}-2), 88.9$ (C-4), 92.1 (C-1), 115.2 (C-5', C-8'), 123.6 (C-6', C-7'), 140.5 (C-4', C-9'), 149.9 (C-2'); HRMS $\left(\mathrm{ESI}^{+}\right) \mathrm{m} / \mathrm{z}$ calcd for $\mathrm{C}_{13} \mathrm{H}_{16} \mathrm{~N}_{2} \mathrm{NaO}_{4} \mathrm{~S}[\mathrm{M}+\mathrm{Na}]^{+}$319.0729, found, 319.0729.

Ammonium D-Glucofuranosyl 1-Phosphate (27). This compound was prepared as described in general procedure $\mathrm{C}$ starting from donor $12(59 \mathrm{mg}, 0.19 \mathrm{mmol})$. After stirring for $90 \mathrm{~min}$ and workup, an amorphous colorless solid $(27 \mathrm{mg})$ containing an anomeric mixture of $\mathbf{2 7}(\alpha / \beta, 1.9: 1 ; 48 \%), \mathbf{2 8}(3 \%)$, and glucose (1\%) was then isolated. $27 \alpha$ : ${ }^{1} \mathrm{H}$ NMR $(500 \mathrm{MHz}$, $\left.\mathrm{D}_{2} \mathrm{O}\right) \delta 3.66(\mathrm{dd}, 1 \mathrm{H}, J=5.6,12.0 \mathrm{~Hz}, \mathrm{H}-6 \mathrm{a}), 3.74(\mathrm{dd}, 1 \mathrm{H}, J$ $=2.8,12.0 \mathrm{~Hz}, \mathrm{H}-6 \mathrm{~b}$ ), 3.80-3.85 (ddd, $1 \mathrm{H}, J=2.8,5.6,8.4$ $\mathrm{Hz}, \mathrm{H}-5$ ), 4.10 (dd, $1 \mathrm{H}, J=2.6,4.1 \mathrm{~Hz}, \mathrm{H}-2$ ), 4.14 (dd, $1 \mathrm{H}, J$ $=4.3,8.4 \mathrm{~Hz}, \mathrm{H}-4), 4.26(\mathrm{dd}, 1 \mathrm{H}, J=2.6,4.3 \mathrm{~Hz}, \mathrm{H}-3), 5.65$ $(\mathrm{dd}, 1 \mathrm{H}, J=4.1,6.4 \mathrm{~Hz}, \mathrm{H}-1) ;{ }^{13} \mathrm{C} \mathrm{NMR}\left(125 \mathrm{MHz}, \mathrm{D}_{2} \mathrm{O}\right) \delta$ 62.7 (C-6), 69.5 (C-5), 75.5 (C-3), 77.3 (d, $J=4.8 \mathrm{~Hz}, \mathrm{C}-2), 78.3$ (C-4), $98.0(\mathrm{~d}, J=3.2 \mathrm{~Hz}, \mathrm{C}-1) ;{ }^{31} \mathrm{P}$ NMR $\left(162 \mathrm{MHz}, \mathrm{D}_{2} \mathrm{O}\right) \delta$ 3.3. $27 \beta$ : ${ }^{1} \mathrm{H}$ NMR $\left(500 \mathrm{MHz}, \mathrm{D}_{2} \mathrm{O}\right) \delta 3.66(\mathrm{dd}, 1 \mathrm{H}, J=5.6$, $12.5 \mathrm{~Hz}, \mathrm{H}-6 \mathrm{a}$ ), 3.79 (dd, $1 \mathrm{H}, J=2.8,12.5 \mathrm{~Hz}, \mathrm{H}-6 \mathrm{~b}$ ), 4.02 (ddd, $1 \mathrm{H}, J=2.8,5.6,8.4 \mathrm{~Hz}, \mathrm{H}-5), 4.08(\mathrm{dd}, 1 \mathrm{H}, J=4.3,8.4 \mathrm{~Hz}$, $\mathrm{H}-4), 4.17(\mathrm{~d}, 1 \mathrm{H}, J=4.3 \mathrm{~Hz}, \mathrm{H}-3), 4.19(\mathrm{br} \mathrm{s}, 1 \mathrm{H}, \mathrm{H}-2), 5.39$ $(\mathrm{d}, 1 \mathrm{H}, J=7.1 \mathrm{~Hz}, \mathrm{H}-1) ;{ }^{13} \mathrm{C}$ NMR $\left(125 \mathrm{MHz}, \mathrm{D}_{2} \mathrm{O}\right) \delta 63.3$ (C-6), 70.0 (C-5), 75.3 (C-3), 80.8 (d, $J=6.5 \mathrm{~Hz}, \mathrm{C}-2), 81.5$ (C-4), 103.3 (d, $J=4.0 \mathrm{~Hz}, \mathrm{C}-1) ;{ }^{31} \mathrm{P}$ NMR $\left(162 \mathrm{MHz}, \mathrm{D}_{2} \mathrm{O}\right) \delta$ 2.6; HRMS $\left(\mathrm{ESI}^{-}\right) \mathrm{m} / z$ calcd for $\mathrm{C}_{6} \mathrm{H}_{12} \mathrm{O}_{9} \mathrm{P}\left[\mathrm{M}-\mathrm{NH}_{4}-\mathrm{NH}_{3}\right]^{-}$ 259.0219 , found 259.0215 . 
Ammonium D-Mannofuranosyl 1-Phosphate (29). This compound was synthesized according to the general procedure C from 15 (54 mg, $0.17 \mathrm{mmol}$ ) after stirring for $15 \mathrm{~min}$. An anomeric mixture of $\mathbf{2 9}(\alpha / \beta, 1.6: 1)$ was then isolated as an amorphous colorless solid (32 mg, 67\%). 29 $\alpha$ : ${ }^{1} \mathrm{H}$ NMR (500 $\left.\mathrm{MHz}, \mathrm{D}_{2} \mathrm{O}\right) \delta 3.59(\mathrm{dd}, 1 \mathrm{H}, J=5.2,12.2 \mathrm{~Hz}, \mathrm{H}-6 \mathrm{a}), 3.66(\mathrm{dd}$, $1 \mathrm{H}, J=2.9,12.2 \mathrm{~Hz}, \mathrm{H}-6 \mathrm{~b}$ ), 3.81 (ddd, $1 \mathrm{H}, J=2.9,5.2,8.7$ $\mathrm{Hz}, \mathrm{H}-5), 4.03-4.06$ (m, 1H, H-4), 4.09 (t, 1H, $J=4.1 \mathrm{~Hz}, \mathrm{H}-2)$, 4.29 (t, $1 \mathrm{H}, J=4.1 \mathrm{~Hz}, \mathrm{H}-3), 5.36$ (dd, $1 \mathrm{H}, J=3.8,6.1 \mathrm{~Hz}$, $\mathrm{H}-1) ;{ }^{13} \mathrm{C}$ NMR $\left(125 \mathrm{MHz}, \mathrm{D}_{2} \mathrm{O}\right) \delta 63.1(\mathrm{C}-6), 69.4(\mathrm{C}-5), 71.2$ (C-3), 78.1 (d, $J=7.2 \mathrm{~Hz}, \mathrm{C}-2$ ), 79.7 (C-4), 102.7 (d, $J=4.8$ $\mathrm{Hz}, \mathrm{C}-1) ;{ }^{31} \mathrm{P}$ NMR $\left(162 \mathrm{MHz}, \mathrm{D}_{2} \mathrm{O}\right) \delta 3.0 .29 \beta:{ }^{1} \mathrm{H}$ NMR $(500$ $\left.\mathrm{MHz}, \mathrm{D}_{2} \mathrm{O}\right) \delta 3.60$ (dd, $1 \mathrm{H}, J=5.8,11.9 \mathrm{~Hz}, \mathrm{H}-6 \mathrm{a}$ ), 3.74 (dd, $1 \mathrm{H}, J=3.0,11.9 \mathrm{~Hz}, \mathrm{H}-6 \mathrm{~b}), 3.85(\mathrm{dd}, 1 \mathrm{H}, J=4.1,8.3 \mathrm{~Hz}$, H-4), 3.95 (ddd, $1 \mathrm{H}, J=3.0,5.8,8.3 \mathrm{~Hz}, \mathrm{H}-5), 4.03-4.06$ (m, $1 \mathrm{H}, \mathrm{H}-2), 4.14$ (t, $1 \mathrm{H}, J=4.7 \mathrm{~Hz}, \mathrm{H}-3), 5.41$ (dd, $1 \mathrm{H}, J=4.8$, $6.4 \mathrm{~Hz}, \mathrm{H}-1) ;{ }^{13} \mathrm{C}$ NMR (125 MHz, $\left.\mathrm{D}_{2} \mathrm{O}\right) \delta 63.1$ (C-6), 70.4 (C-5), 70.5 (C-3), 72.5 (d, $J=6.4 \mathrm{~Hz}, \mathrm{C}-2), 79.7$ (C-4), 96.5 (d, $J=4.8 \mathrm{~Hz}, \mathrm{C}-1) ;{ }^{31} \mathrm{P} \mathrm{NMR}\left(162 \mathrm{MHz}, \mathrm{D}_{2} \mathrm{O}\right) \delta 3.0 ;$ HRMS $\left(\mathrm{ESI}^{-}\right)$ $m / z$ calcd for $\mathrm{C}_{6} \mathrm{H}_{12} \mathrm{O}_{9} \mathrm{P}\left[\mathrm{M}-\mathrm{NH}_{4}-\mathrm{NH}_{3}\right]^{-} 259.0219$, found 259.0207.

Ammonium D-Fucofuranosyl 1-Phosphate (30). This compound was prepared according to the general procedure C from 26 (50 mg, $0.16 \mathrm{mmol}$ ) after stirring for $20 \mathrm{~min}$. An anomeric mixture of $\mathbf{3 0}(\alpha / \beta, 1.5: 1)$ was isolated as an amorphous colorless solid (42 mg, 90\%). 30 $\alpha$ : ${ }^{1} \mathrm{H} \mathrm{NMR} \mathrm{(500} \mathrm{MHz,}$ $\left.\mathrm{D}_{2} \mathrm{O}\right) \delta 1.11(\mathrm{~d}, 3 \mathrm{H}, J=6.4 \mathrm{~Hz}, \mathrm{H}-6), 3.45-3.49(\mathrm{~m}, 1 \mathrm{H}, \mathrm{H}-4)$, 3.76-3.84 (m, 1H, H-5), 3.95-4.01 (m, 2H, H-2, H-3), 5.39 (dd, $1 \mathrm{H}, J=3.8,5.1 \mathrm{~Hz}, \mathrm{H}-1) ;{ }^{13} \mathrm{C}$ NMR $\left(125 \mathrm{MHz}, \mathrm{D}_{2} \mathrm{O}\right) \delta 17.8$
(C-6), 69.2 (C-5), 75.4 (C-3), 77.7 (d, $J=7.2 \mathrm{~Hz}, \mathrm{C}-2), 85.9$ (C4), $96.4(\mathrm{~d}, J=5.6 \mathrm{~Hz}, \mathrm{C}-1) ;{ }^{31} \mathrm{P}$ NMR $\left(162 \mathrm{MHz}, \mathrm{D}_{2} \mathrm{O}\right) \delta 3.1$. $30 \beta$ : ${ }^{1} \mathrm{H}$ NMR $\left(500 \mathrm{MHz}, \mathrm{D}_{2} \mathrm{O}\right) \delta 1.16(\mathrm{~d}, 3 \mathrm{H}, J=5.8 \mathrm{~Hz}, \mathrm{H}-6)$, 3.76-3.84 (m, 3H, H-3, H-4, H-5), 4.06 (br s, 1H, H-2), 5.36 $(\mathrm{d}, 3 \mathrm{H}, J=6.4 \mathrm{~Hz}, \mathrm{H}-1) ;{ }^{13} \mathrm{C}$ NMR $\left(125 \mathrm{MHz}, \mathrm{D}_{2} \mathrm{O}\right) \delta 18.3(\mathrm{C}-$ 6), 68.2 (C-5), 77.6 (C-3), 82.2 (d, $J=7.2 \mathrm{~Hz}, \mathrm{C}-2), 88.1$ (C-4), $102.6(\mathrm{~d}, J=4.0 \mathrm{~Hz}, \mathrm{C}-1)$; ${ }^{1} \mathrm{P}$ NMR $\left(162 \mathrm{MHz}, \mathrm{D}_{2} \mathrm{O}\right) \delta 2.5$; HRMS $\left(\mathrm{ESI}^{-}\right) \mathrm{m} / \mathrm{z}$ calcd for $\mathrm{C}_{6} \mathrm{H}_{12} \mathrm{O}_{8} \mathrm{P}\left[\mathrm{M}-\mathrm{NH}_{4}-\mathrm{NH}_{3}\right]^{-}$ 243.0270 , found 243.0274 .

Acknowledgment. We are grateful to the French Ministry of Education, Research, and Technology for a grant to R.E. We also thank Martine Lefeuvre and JeanPaul Guégan (ENSCR) and Sourisak Sinbandhit (Centre Régional de Mesures Physiques de l'Ouest, University of Rennes 1, France) for recording NMR spectra and Serge Pilard (University of Amiens, France) for recording mass spectra. We are finally grateful to Françoise Chrétien and Yves Chapleur (University of Nancy) for helpful discussions.

Supporting Information Available: One-dimensional and two-dimensional correlational NMR spectra, ${ }^{1} \mathrm{H}-{ }^{1} \mathrm{H}$ and ${ }^{1} \mathrm{H}-{ }^{13} \mathrm{C}$ for all products. This material is available free of charge via the Internet at http://pubs.acs.org.

JO0484934 